\title{
Identifying resonances of the Galactic bar in Gaia DR2: II. Clues from angle space
}

\author{
Wilma H. Trick ${ }^{1 \star}$ \\ ${ }^{1}$ Max-Planck-Insitut für Astrophysik, Karl-Schwarzschild-Str. 1, D-85748 Garching b. München, Germany
}

Accepted XXX. Received YYY; in original form ZZZ

\begin{abstract}
The Milky Way disk exhibits intricate orbit substructure of still-debated dynamical origin. The angle variables $\left(\theta_{\phi}, \theta_{R}\right)$ - which are conjugates to the actions $\left(L_{z}, J_{R}\right)$, and describe a star's location along its orbit-are a powerful diagnostic to identify $l: m$ resonances via the orbit shape relation $\Delta \theta_{R} / \Delta \theta_{\phi}=-m / l$. In the past, angle signatures have been hidden by survey selection effects (SEs). Using test particle simulations of a barred galaxy, we demonstrate that Gaia should allow us to identify the Galactic bar's Outer Lindblad Resonance $(l=+1, m=2$, OLR) in angle space. We investigate strategies to overcome SEs. In the angle data of the Gaia DR2 RVS sample, we independently identify four candidates for the OLR and therefore for the pattern speed $\Omega_{\mathrm{bar}}$. The strongest candidate, $\Omega_{\mathrm{bar}} \sim 1.4 \Omega_{0}$, positions the OLR above the 'Sirius' moving group, agrees with measurements from the Galactic center, and might be supported by higher-order resonances around the 'Hercules/Horn'. But it misses the classic orbit orientation flip, as discussed in the companion study on actions. The candidate $\Omega_{\text {bar }} \sim 1.2 \Omega_{0}$ was also suggested by the action-based study, has the OLR at the 'Hat', is consistent with slow bar models, but still affected by SEs. Weaker candidates are $\Omega_{\text {bar }}=1.6 \Omega_{0}$ and $1.74 \Omega_{0}$. In addition, we show that the stellar angles do not support the 'Hercules/Horn' being created by the OLR of a fast bar. We conclude that - to resolve if 'Sirius' or 'Hat' is related to the bar's OLR-more complex dynamical explanations and more extended data with well-behaved SEs are required.
\end{abstract}

Key words: Galaxy: disc - Galaxy: kinematics and dynamics

\section{INTRODUCTION}

Two puzzles have engaged Galactic astronomers for over two decades, and an unambiguous answer would be a milestone in understanding the mechanisms that have shaped our Milky Way (MW):

(1) What is the value of the Galactic bar's pattern speed, $\Omega_{\mathrm{bar}}$ ?

(2) Which features observed in the local stellar phase-space are due to bar resonances?

These two aspects of the Galactic structure are coupled via the resonance condition

$m \cdot\left(\Omega_{\phi, \text { true }}-\Omega_{\text {bar }}\right)+l \cdot \Omega_{R, \text { true }}=0$,

(where $\Omega_{\phi \text {,true }}$ and $\Omega_{R \text {,true }}$ are the primary azimuthal and radial frequency of a stellar orbit, and $l, m \in \mathbb{Z}$ ), and via the Galaxy's gravitational potential $\Phi(\mathbf{x})$, in particular:

(i) the rotation curve $v_{\text {circ }}(R \mid \Phi)$ which sets the orbital frequencies and therefore the location of the bar resonances in the disk,

(ii) the mass and shape of the bar which set the strength and

^ E-mail: trick@mpa-garching.mpg.de number of the local resonance features (e.g. Hunt \& Bovy 2018; Monari et al. 2019a),

(iii) additional mechanisms that might affect and obscure the bar resonances (e.g. spiral arms (Quillen 2003; Minchev \& Famaey 2010; Quillen et al. 2011; Monari et al. 2016; Fujii et al. 2019; Hunt et al. 2019; Pettitt et al. 2020), satellite interaction (Khanna et al. 2019; Laporte et al. 2019), or bar deceleration (Chiba et al. 2021)).

The above questions (1) and (2) have been studied in the past together or independently, using different approaches and data sets.

Recent studies measured bar pattern speeds around $\Omega_{\text {bar }} \sim$ $40 \mathrm{~km} / \mathrm{s} / \mathrm{kpc}$ directly, e.g. from Gaia proper motions (Gaia Collaboration et al. 2016) observed in the Galactic center (Sanders et al. 2019; Clarke et al. 2019; Bovy et al. 2019) using the Tremaine \& Weinberg (1984) method, from made-to-measure modeling of red clump stars (Portail et al. 2017), or interpreting observed gas flows (Sormani et al. 2015).

The 'Hercules' stream is a prominent feature in the stellar velocities within $\sim 200 \mathrm{pc}$ from the Sun at $V \sim-50 \mathrm{~km} / \mathrm{s}$. It has been noted to resemble the expected signature of the 1:2 Outer Lindblad Resonance ( $l=+1, m=2$, OLR for short) of a short fast bar (e.g. Dehnen 2000; Fux 2001a; Antoja et al. 2014; Monari et al. $2017 b)$, of the Co-rotation Resonance $(l=0, \mathrm{CR})$ of a long slow 
bar (e.g. Pérez-Villegas et al. 2017; Monari et al. 2019b; Binney 2020b; D'Onghia \& L. Aguerri 2020), or of the outer 1:4 Lindblad resonance $(l=+1, m=4)$ of a slightly faster slow bar (Hunt \& Bovy 2018).

The precision and accuracy of the stellar velocities measured by the Gaia satellite's second data release (DR2, Gaia Collaboration et al. 2018a; Katz et al. 2019) have made local moving groups visible in unprecedented detail (Gaia Collaboration et al. 2018b). Several authors have noted that the 'Hat' moving group at $V \sim 40 \mathrm{~km} / \mathrm{s}$ could look like an OLR also-in this case caused by a slow bar (Monari et al. 2019b; Hunt et al. 2019; Trick et al. 2021).

The large spatial extent of Gaia DR2's 6D stellar phase-space data has spiked the interest in studying the features in the disk's orbit distribution and bar resonances in the disk beyond the immediate Solar neighbourhood. These orbit features are prevalent over a few kpc from the Sun and project locally into the classic moving groups (Trick et al. 2019, T19 hereafter). They have been studied in the $\left(R, v_{\phi}\right)$ plane (Kawata et al. 2018), where the OLR creates elongated arches (e.g., Fragkoudi et al. 2019), the $\left(L_{z}, \phi\right)$ plane, where the sloping of the orbit structures could be informative about the resonances (e.g., Monari et al. 2019c; D'Onghia \& L. Aguerri 2020; Friske \& Schönrich 2019; Chiba et al. 2021), and action space $\left(L_{z}, J_{R}\right)$ (T19), where resonances create high- $J_{R}$ ridges (e.g., Sellwood 2010; Sellwood et al. 2019; Monari et al. 2019a; Hunt et al. 2019; Trick et al. 2021).

The discrepancy between the slow bar model from the Galactic center modeling and the fast bar from the 'Hercules' stream modeling is a classic conundrum of Galactic dynamics. Recently, a new inconsistency has emerged: The newest Galactic center measurements of the bar pattern speed do not position their OLR close to any plausible feature in the local kinematics. Monari et al. (2019a) mentions that the slow bar by Portail et al. (2017) and Pérez-Villegas et al. (2017) requires modifications to the MW's assumed rotation curve or Solar motion (e.g. Schönrich et al. 2010) to consolidate the OLR with the 'Hat' feature (at $V \sim 40 \mathrm{~km} / \mathrm{s}$ ). Hunt et al. (2019) also noticed that for the slow bar pattern speed, the resonant ridges fall in the wrong phase-space locations to agree with the Gaia data. In Trick et al. (2021), we showed that by combining the slightly faster slow bar $\Omega_{\text {bar }}$ by Sanders et al. (2019) and Bovy et al. (2019) with recent models for the MW's rotation curve (Bovy 2015; Eilers et al. 2019), the OLR falls close to the Sirius ridges (with the associated 'Sirius' moving group being at $(U, V)=(10,3) \mathrm{km} / \mathrm{s})$, a region in phase-space that does not agree with the naive expectation for an OLR signature.

Several intriguing proposals could resolve this new contradiction. (A) Chiba et al. (2021) have studied a decelerating bar model-as opposed to the often used bar models with a constant pattern speed-which creates signatures that could indeed be closer to the observed ones around the 'Sirius' moving group with the current OLR at the 'Hat'. (B) Galaxy simulations suggest that in more complex environments, the most reliable signature of the OLR is the high- $J_{R}$ ridge it creates (Fragkoudi et al. 2020; Kawata et al. 2021), as spiral arms might obscure the idealized $U \sim v_{R}$ pattern of the OLR (Fujii et al. 2019). (C) Spiral arm models can create ridges just as strong as those observed in the Gaia data (e.g., De Simone et al. 2004; Antoja et al. 2009; Sellwood et al. 2019; Hunt et al. 2019). Work by Quillen et al. (2018) and Khoperskov et al. (2020) associates the main features we see in the local data not with the bar resonances, but with the MW's spiral arms as identified by Reid et al. (2014) and Xu et al. (2016): the 'Hat' to the Perseus arm (see also Hunt et al. 2017), 'Sirius' to the Local Arm, the 'Horn' (at $(U, V) \sim(50,-20) \mathrm{km} / \mathrm{s})$ to the Sagittarius arm, and 'Hercules' to the Scutum arm. (D) Our knowledge of the MW's gravitational potential and the Sun's motion might be the culprit (Monari et al. 2017a, 2019a).

In summary, the complexity and degeneracy of all the mechanisms possibly at play in the Galactic disk call for (a) a deeper understanding of the effect different mechanisms can have alone and together, and (b) straightforward, discriminating diagnostics that help to determine which mechanism causes which orbital feature in the Gaia data. Extending our investigations in Trick et al. (2021) (Paper I, hereafter), we focus in this work therefore on (a) bar resonances in a basic MW model (a galactic disk, perturbed by a boxy bar with a constant pattern speed and without spiral arms), and study (b) the signatures caused in the space of orbital phase angles. The radial phase $\theta_{R}$ and the azimuthal phase $\theta_{\phi}$ describe the location of a star on its orbit. They are canonical conjugates to the orbital actions $J_{R}$ and $J_{\phi}=L_{z}$, respectively. Their physical interpretation is illustrated in Figure 1.

The diagnostic power of phase-angles has previously been noted in the literature. Sellwood (2010) showed that the 'Hyades' moving group (around $(U, V)=(-33,-16) \mathrm{km} / \mathrm{s})$ is consistent with being created by an inner $(l=-1)$ Lindblad resonance, based on the idea that angle-space can be used to identify the exact $(l, m)$ of a resonant feature. Follow-up studies by McMillan (2011) and Hahn et al. (2011) agreed with Sellwood (2010) that action-space indeed supports the resonant origin of the 'Hyades', which is also in concordance with age-abundance measurements (Famaey et al. 2007; Pompéia et al. 2011). However, they pointed out that for the $d<200$ pc survey volume of the pre-Gaia era, selection effects in angle-space are too severe to unambiguously determine the $(l, m)$ of the resonance at the 'Hyades'. They all agreed that a larger coverage of data in the Galactic disk might help with disentangling physical signatures from selection biases in angle space. In Section 5.4, we will discuss the work by Sellwood (2010) and McMillan (2011) in more detail, in the context of comparing to our results.

The exquisite data quality of Gaia DR2 motivated Sellwood et al. (2019) and Hunt et al. (2019) to revisit the Solar neighbourhood $d<200 \mathrm{pc}$ in angle space, making the intricate disk substructure beyond the 'Hyades' visible. As pointed out in fig. 3 in Hunt et al. (2019) (and as will be discussed in Section 3.2), this provides an alternative way to show the well-known moving groups.

This paper is structured as follows. In Section 2, we revisit resonant orbits in action-angle space. Inspired by the study of Sellwood (2010), we present in Sections 3.1-3.2 a method based on angle-space to identify the bar OLR directly from the Gaia data. Cautioned by the study of McMillan (2011), we develop a strategy to disentangle physical signatures from selection effects in Sections 3.3-3.4, even though we do not know Gaia's detailed completeness function. In Section 4.1-4.2, we search for the bar's OLR in the in-plane angle space of the Gaia DR2 radial velocity sample (RVS) (Katz et al. 2019) out to $d=3 \mathrm{kpc}$, and present our favoured candidates for the corresponding bar pattern speed $\Omega_{\mathrm{bar}}$. In the discussion, Section 5, we compare these candidates with the OLR candidates found from the method based on action- $v_{R}$-space in Paper I, present a curious coincidence related to higher-order resonances, and discuss previous work. For detailed discussions of the bar pattern speed and OLR candidates in the literature, we refer the reader to Paper I. We summarize in Section 6. 


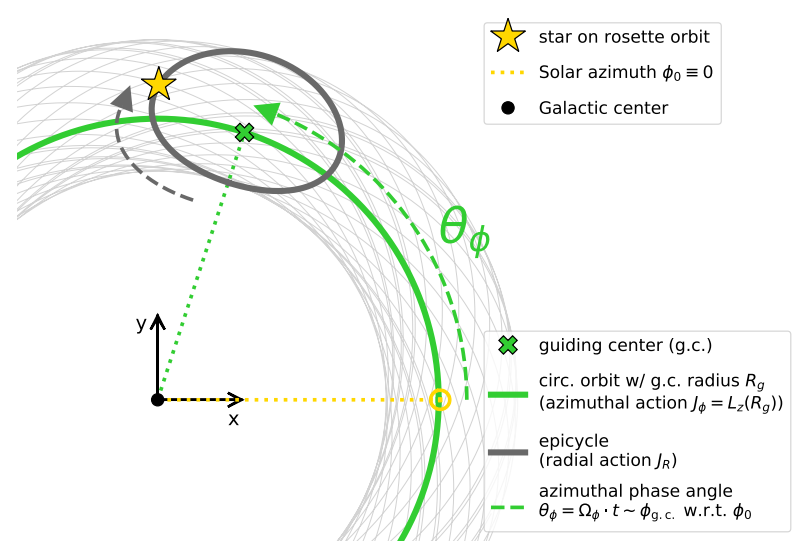

(a) The azimuthal angle $\theta_{\phi}$ is the azimuth of the guiding center.

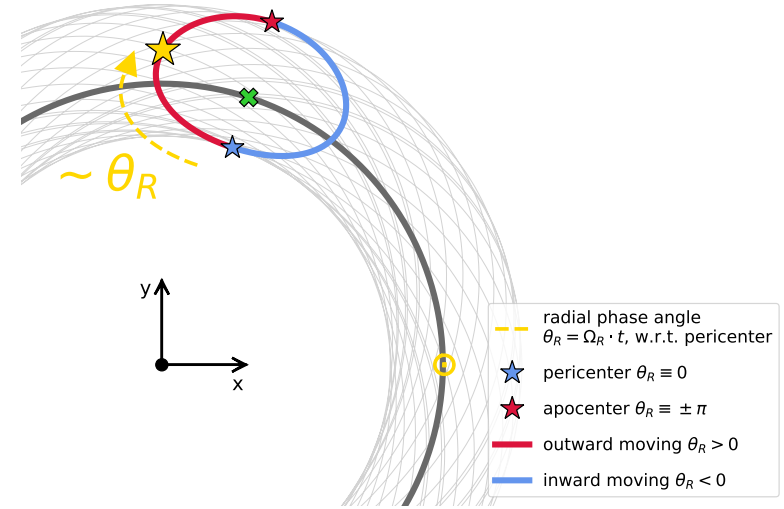

(b) The radial angle $\theta_{R}$ describes the radial phase between peri- and apocenter.

Figure 1. Illustration of how to interpret the in-plane phase angles $\theta_{\phi}$ and $\theta_{R}$ associated with the actions $J_{\phi} \equiv L_{z}$ and $J_{R}$ in the epicycle approximation for a disk orbit in an axisymmetric galaxy disk.

\section{RESONANT ORBITS IN ANGLE SPACE}

\subsection{The coordinates: Axisymmetric action-angle estimates}

A star on a near-circular in-plane orbit in the Galactic disk follows a rosette-like path. Its motion can be decomposed into a circular orbit with angular momentum $L_{z}$ and a radial epicycle motion (Figure 1). The star moves along the epicycle around the guiding center with the radial frequency $\Omega_{R}$; the guiding center moves around the Galactic center with the tangential frequency $\Omega_{\phi}$. The size of the circular orbit is related to the azimuthal action

$J_{\phi} \equiv L_{z} \equiv R_{g} \times v_{\text {circ }}\left(R_{g}\right)=R \cdot v_{\phi}$,

where $R_{g}$ is the guiding center radius, $v_{\text {circ }}^{2}=R \times \partial \Phi /\left.\partial R\right|_{z=0}$, $(R, z, \phi)$ Galactocentric cylindrical coordinates, and $\left(v_{R}, v_{\phi}, v_{z}\right)$ the velocities in the corresponding directions. The size of the epicycle is related to the radial action

$J_{R} \equiv \frac{1}{\pi} \int_{u_{\min }}^{u_{\max }} p_{u} \mathrm{~d} u \in[0, \infty)$.

In the prolate confocal coordinate system $(u, v, \phi), u$ is constant on ellipses in the $(R, z)$ plane. In the plane $(z=0), u$ is therefore a radius-like coordinate (Binney \& Tremaine 2008, §3.5.3). In an axisymmetric gravitational galaxy potential, these two actions are conserved canonical momenta whose conjugate positions are the phase angles $\theta_{\phi}$ and $\theta_{R}$. Action-angles have convenient properties:

$$
\begin{aligned}
& \dot{j}_{i}=-\frac{\partial \mathscr{H}_{\mathrm{axi}}}{\partial \theta_{i}}=0 \quad \Longrightarrow \quad J_{i}(t)=\text { const. } \\
& \dot{\theta}_{i}=\frac{\partial \mathscr{H}_{\mathrm{axi}}}{\partial J_{i}}=\Omega_{i, \mathrm{axi}}(\mathbf{J}) \quad \Longrightarrow \quad \theta_{i}(t)=\Omega_{i, \mathrm{axi}} \cdot t+\theta_{i, 0},
\end{aligned}
$$

with $i \in\{R, \phi, z\}, \mathbf{J}=\left(J_{R}, J_{\phi}, J_{z}\right)$, and where $\mathscr{H}_{\text {axi }}$ is the Hamiltonian of the axisymmetric system (Binney \& Tremaine 2008, $\S 3.5)$. $(\theta, \mathbf{J})$ describes the same full $6 \mathrm{D}$ phase-space as the usual position-velocity space $(\mathbf{x}, \mathbf{v})$. The angles increase linearly, $\mathbf{x}$ and $\mathbf{v}$ are periodic in the angles, and in this work, we define the angles to be $\theta_{i} \in[-\pi, \pi]$.. The angle $\theta_{R}$ can be interpreted as the current radial phase position of the star along the epicycle between periand apocenter, with respect to the pericenter at $\theta_{R} \equiv 0$ (Figure 1(b)). The angle $\theta_{\phi}$ is the current polar azimuth $\phi$ of the guiding center with respect to the azimuth of the Sun today, i.e. $\phi_{0} \equiv 0$ (Figure 1(a)). ${ }^{1}$ A nice explanation of the angles can also be found in McMillan (2011, §2).

To calculate these actions, angles, and frequencies, one requires measurements of a star's current position $\mathbf{x}$ and velocity $\mathbf{v}$ (e.g. by Gaia Collaboration et al. 2016), as well as an assumption for the axisymmetric gravitational galaxy potential in which the star moves (e.g. the MWPotential2014 by Bovy 2015), and an efficient estimation algorithm (e.g. the Stäckel fudge by Binney 2012; Bovy \& Rix 2013).

Naturally, the Galaxy is not axisymmetric, as it has-among other perturbing mechanisms - also a strong central bar which rotates with a pattern speed $\Omega_{\mathrm{bar}}$. In this case, actions and angles calculated in the axisymmetric background potential at each point in time still describe coordinates for the stars as valid as positions and velocities. The actions $\left(J_{R}, L_{z}\right)$ are, however, not fully conserved anymore (Equation 4), and the angles $\left(\theta_{R}, \theta_{\phi}\right)$ do not follow the exact linear relation in Equation (5). As shown in Trick et al. (2021), these axisymmetric action estimates are still very informative about the perturbed system: In particular the axisymmetric resonance lines $(A R L s)$, i.e. the lines in axisymmetric action space along which the resonance condition is satisfied,

$m \cdot\left(\Omega_{\phi, \text { axi }}-\Omega_{\text {bar }}\right)+l \cdot \Omega_{R, \text { axi }}=0$

(with $l, m \in \mathbb{Z}$, and $J_{z}=0$ ), prove to be helpful diagnostic tools.

In this work, we distinguish between two sets of orbital frequencies:

- $\Omega_{R \text {,true }}$ and $\Omega_{\phi \text {,true }}$ are the real radial and azimuthal orbital frequencies in a barred system. They are determined as the primary frequencies from a Fourier transform of the actual time-evolved orbit in the barred potential (see Section 3.1 and Figure A1).

- $\Omega_{R \text {,axi }}$ and $\Omega_{\phi \text {,axi }}$ are the axisymmetric frequency estimates. They are calculated instantaneously in the axisymmetric background potential and are associated with the axisymmetric actionangle estimates in Equation (5).

${ }^{1}$ Note that this straightforward interpretation of actions and angles based on the epicycle approximation is only valid for near-circular disk orbits. 
These frequencies can differ substantially from each other.

\subsection{Background: Orbits in resonance with the bar}

The behaviour in action-angle space of orbits in bar potentials is well studied in the literature. Introductions based on perturbation theory can be found in Monari et al. (2017c) and Chiba et al. (2021), and a more rigorous derivation in Binney (2018). In Paper I, we illustrated the action-space behaviour by means of numerically integrated orbits. Here, we summarize the most important concepts, which are also illustrated for one family of OLR orbits in Figure A1 in Appendix A.

Rosette orbits in axisymmetric galaxy potentials have peri- and apocenters at any possible azimuth $\phi \in[-\pi, \pi]$. Over time, such an orbit will fill the whole $(2 \pi)^{2}$ angle plane. The same is true for non-resonant orbits in barred potentials. This orbit characteristic is called circulation.

Resonant orbits, whose orbital frequencies $\Omega_{i \text {,true }}$ are exactly commensurate with the bar's pattern speed by the ratio $l: m$ according to the resonance condition Equation (1), behave differently. We illustrate this using the axisymmetric action-angle-frequency estimates. The resonance condition for the $\Omega_{i \text {,axi }}$ (Equation 6) is not exactly satisfied, but becomes very small,

$\Omega_{\text {slow }} \equiv m \cdot\left(\Omega_{\phi, \text { axi }}-\Omega_{\text {bar }}\right)+l \cdot \Omega_{R, \text { axi }} \sim 0$

(see, e.g., eq. (11) and (12) in Chiba et al. 2021). By time-integrating Equation (7)—using $\phi_{\text {bar }}(t) \equiv \Omega_{\text {bar }} t+\phi_{\text {bar, } 0}$, and Equation (5)—we get

$\theta_{\text {slow }}(t) \equiv m \cdot\left(\theta_{\phi}(t)-\phi_{\text {bar }}(t)\right)+l \cdot \theta_{R}(t) \sim$ const.,

i.e., an axisymmetric estimate for an angle coordinate that evolves very slowly for an orbit close to the $l: m$ resonance (e.g., Weinberg 1994). The angles $\theta_{R}$ and $\theta_{\phi}$ still evolve fast in time, and the radial phase is often called the fast angle of the resonant motion,

$\theta_{\text {fast }} \equiv \theta_{R}$.

A family of resonant orbits trapped at a given $l: m$ resonance consists of (i) one closed periodic parent orbit and (ii) librating orbits. The orbits in the same resonant orbit family have the same Jacobi energy

$E_{\mathrm{Jacobi}}=E-\Omega_{\mathrm{bar}} \cdot L_{z}=$ const.,

where $E \equiv\left(v_{R}^{2}+v_{\phi}^{2}\right) / 2+\Phi(R, \phi)$. $E_{\mathrm{Jacobi}}$ is a conserved quantity in a triaxial, rotating system (Binney \& Tremaine 2008, eq. 3.112). From this follows directly - using the resonance condition in Equation (6), and $E_{i} \sim J_{i} \Omega_{i, \text { axi }}$ as the energy stored in the motion in the $i$-th coordinate direction - that they also have a similar values of the fast action

$J_{\text {fast }} \equiv J_{R}-\frac{l}{m} L_{z} \sim$ const.

(Lynden-Bell 1979; Kaasalainen 1994; Weinberg 1994). All these orbits oscillate in the $\left(L_{z}, J_{R}\right)$ action plane. The $\theta_{\text {fast }}$-oscillation of the radial phase has only a small amplitude in action space and is in perturbation studies often averaged over.

The parent orbit closes in the frame co-rotating with the bar's pattern speed (CRF) after $l$ rotations around the bar, moving $m$-times inwards and outwards. The $m$ pericenters occur at the azimuthal positions

$\phi_{\mathrm{CRF}, \text { peri }, i} \equiv \phi_{\mathrm{CRF}, \text { peri }, 0}+i \cdot \frac{2 \pi l}{m}, i \in \mathbb{Z}$.
In angle space, the parent orbit follows therefore exactly the relation

$\theta_{R}(t)=-\frac{m}{l} \cdot\left(\theta_{\phi}(t)-\phi_{\text {bar }}(t)-\phi_{\text {CRF,peri, } 0}\right)$.

The parent orbit has a constant $\theta_{\text {slow }}$ and lives close to the $l: m$ ARL in action space. If we define the 0 -th pericenter $\phi_{\mathrm{CRF}, \text { peri, } 0} \in$ $[0,2 \pi l / m]$, then it is related to the slow angle by

$\theta_{\text {slow }}=m \phi_{\mathrm{CRF}, \text { peri, } 0}$.

The slow angle characterizes therefore the orientation of the parent orbit with respect to the bar.

The librating orbits are characterized by exhibiting slow oscillations around their parent orbit's $\theta_{\text {slow }}$. This manifests as an oscillation (i.e. libration) around the parent orbit (i) of the pericenter and apocenter azimuthal locations with respect to the bar, (ii) in action space along a line of constant $J_{\text {fast }}$ with slope

$\frac{\Delta J_{R}}{\Delta L_{z}}=\frac{l}{m}$

and (iii) within a stripe of slope

$\frac{\Delta \theta_{R}}{\Delta\left(\theta_{\phi}-\phi_{\text {bar }}\right)}=-\frac{m}{l}$

around the relation Equation (13) in angle space. The librating orbits differ only in their libration amplitude. The maximum possible libration amplitude depends on the strength of the bar's $m$-th Fourier mode. The boundary at the maximum libration amplitude is called the separatrix. In action space, it is roughly parallel to the ARL on both sides and marks the region within which orbits are trapped at the resonance and librate. Outside, orbits circulate freely.

\subsection{Examples: The angle signature of resonant orbits}

As an example, we show in the upper panels of Figure 2 librating orbits trapped at four different bar resonances: the 1:2 OLR, the 1:1 and 1:4 outer Lindblad resonances (1:1 and 1:4 resonances for short), and CR with $l=0$. In the frame co-rotating with the bar, they exhibit the well-known and distinctive shapes that have been discussed in depth in the literature (e.g., Athanassoula et al. 1983; Contopoulos \& Grosbol 1989; Dehnen 2000; Fux 2001a; Sellwood \& Wilkinson 1993; Harsoula \& Kalapotharakos 2009; Binney 2018; Fragkoudi et al. 2019; Binney 2020a). We integrated these orbits in the Galaxy potential dominated by an idealized quadrupole bar which we will introduce in Section 3.1. For comparison, we show also one non-resonant circulating orbit.

The lower panels in Figure 2 present these orbits in angle space $\left(\theta_{\phi}, \theta_{R}\right)$. If we consider each orbit as the time evolution of a single star, then the $\theta_{\phi}$-coordinate shown on the $x$-axis is actually the co-rotating

$\theta_{\phi, \mathrm{CRF}}(t) \equiv \theta_{\phi}(t)-\phi_{\mathrm{bar}}(t)+25^{\circ}$.

Figure 2 illustrates that our bar potential aligns the peri- and apocenters of resonant orbits along its major and minor axes. The slow angles of our example orbits with $l>0$ oscillate-following Equations (8) and (14) - around $\theta_{\text {slow, OLR }}=\pi, \theta_{\text {slow, } 1: 1}=3 \pi / 2$, and $\theta_{\text {slow } 1: 4}=0$. For more general bar potentials, it depends on the exact form of the potential around which values of $\theta_{\text {slow }}$ orbits will be trapped.

With the Gaia data in mind, we now consider each example orbit in Figure 2 as a sample of many stars distributed along the same orbit and being observed at a single snapshot in time, $t_{\text {today }} \cdot \theta_{\phi}$ on the $x$-axis denotes then current azimuthal angle in the coordinate 

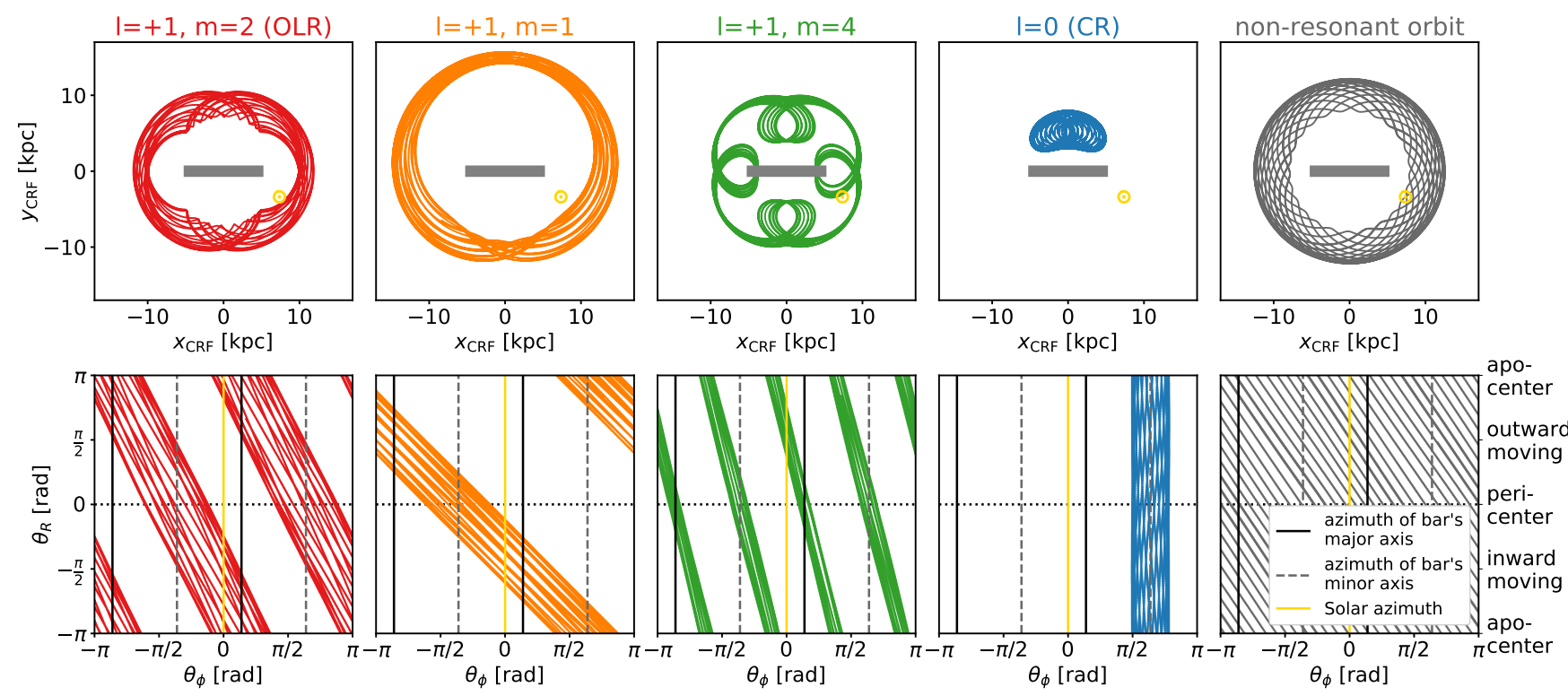

Figure 2. Illustration of five example disk orbits in the Fiducial_40 simulation, trapped at four different $l: m$ bar resonances, and one non-resonant orbit. Upper panels: Time evolution in the Galactic plane $(x, y)$ in the frame co-rotating $(\mathrm{CRF})$ with the bar. Lower panels: The $\left(\theta_{\phi}, \theta_{R}\right)$ angle space, now assuming the orbit was captured at a single snapshot in time and each dot along the orbit was an individual star. The peri- and apocenters of resonant orbits are 'trapped' within a limited azimuthal range with respect to the bar. The distribution in the corresponding angle planes follows $\Delta \theta_{R} / \Delta \theta_{\phi} \sim-m / l$ due to the intrinsic orbit shape.

frame of Figure 1(a), where the Sun is at $\phi_{0}=0$ and the bar at $\phi_{\text {bar }}=+25^{\circ}$.

As a direct consequence of the libration around the parent orbit (Equation 8), the stars on these orbits lie within stripes of slope

$\frac{\Delta \theta_{R}}{\Delta \theta_{\phi}}=-\frac{m}{l}$.

We expect that at the bar resonances many stars on a range of orbits accumulate along specific values of $\theta_{\text {slow }}$ and therefore these characteristic slopes. The reason is that (i) the bar creates a potential trough aligned with the $m$-th bar component's axes within which the resonant stars librate such that discrete values of $\theta_{\text {slow }}$ are preferred (e.g. Binney \& Spergel 1982) and (ii) the trapping regions at bar resonances (especially those with $m=2$ ) cover substantial regions of stellar phase-space (Binney 2018) trapping many stars. At the heart of this study-and also the works of Sellwood (2010) and McMillan (2011) — is therefore the idea that by searching for stellar overdensities in angle space along slopes of $-m / l$, we hope to identify the bar's $l: m$ resonances.

\section{AN ANGLE-BASED METHOD TO IDENTIFY THE OLR}

\subsection{The test particle simulation}

To illustrate and test this idea to identify bar resonances in Gaia's angle space, we have run two test particle simulations similar to those in Trick et al. (2021, see their appendix C for details). An axisymmetric stellar disk with 10 Million particles is generated from the disk distribution function by Binney \& McMillan (2011) in the axisymmetric MWPotential2014 by Bovy (2015) $\left(R_{0} \equiv 8 \mathrm{kpc}, v_{0} \equiv\right.$ $v_{\text {circ }}\left(R_{0}\right)=220 \mathrm{~km} / \mathrm{s}, L_{z, 0} \equiv R_{0} \cdot v_{0}=1760 \mathrm{kpc} \mathrm{km} / \mathrm{s}, \Omega_{0} \equiv$ $v_{0} / R_{0}=27.5 \mathrm{~km} / \mathrm{s} / \mathrm{kpc}$ ). The pure quadrupole $3 \mathrm{D}$ bar model by Dehnen (2000) and Monari et al. (2016) is superimposed onto the background MW potential. In addition, we implemented for a $m=4$ bar component (following eq. (4) in Hunt \& Bovy 2018) an analogous 3D version (following eq. (1) in Monari et al. 2016). As definition for the strength of the different bar components, we use $\alpha_{m}$, which describes the radial force ratio at $R=R_{0}$ and $\phi=\phi_{\text {bar }}$ of the $m$-th Fourier term of the bar model vs. the axisymmetric background model. Analogously to the 2D bar model by Hunt \& Bovy (2018), we build a 2-component bar model with $\alpha_{m=2}=0.01$ and a weak $\alpha_{m=4}=-0.0005$. The latter makes the bar's face-on shape boxy and therefore more realistic. ${ }^{2}$ The bar model has a length of $R_{\mathrm{bar}}=4.5 \mathrm{kpc}$ or $3.5 \mathrm{kpc}$, and rotates with a pattern speed of $\Omega_{\mathrm{bar}}=40 \mathrm{~km} / \mathrm{s} / \mathrm{kpc}$ or $51 \mathrm{~km} / \mathrm{s} / \mathrm{kpc}$, respectively. We refer to these as the Fiducial_40 and the Fiducial_51 simulations. We slowly grow the bar strength from 0 to its final value over 5 bar periods $T_{\text {bar }} \equiv 2 \pi / \Omega_{\text {bar }}$. All particle orbits are integrated in this barred galaxy potential for $T_{\mathrm{int}}=50 T_{\mathrm{bar}}$ using galpy ${ }^{3}$. The azimuth of the bar's major axis in the simulation is

$\phi_{\text {bar }}=\phi_{\text {bar }, 0}+\Omega_{\text {bar }} t, \quad \phi_{\text {bar }, 0} \equiv 25^{\circ}$.

After each full period of orbit integration it is therefore similar to today's configuration between the MW's bar and the Sun, where $\phi_{0}=0$. Snapshots at $t=25 T_{\mathrm{bar}}$ and $t=50 T_{\mathrm{bar}}$ are stacked to increase the number of particles to 20 Million. To this final sample, we apply cuts $L_{z} \in[0.4,1.5] L_{z, 0}$ and $J_{z}<0.01 L_{z, 0}$.

The simulations contain only stars on loop disk orbits that

2 Bars with pronounced non-zero $m=4$ Fourier components have been investigated in simulations (e.g., by Combes \& Sanders 1981; Pfenniger \& Friedli 1991; Athanassoula \& Misiriotis 2002; Hunt \& Bovy 2018), and in the dynamical MW bar model by Portail et al. (2017). Observationally, there are indications that boxy bars might occur more often in late-type disk galaxies, and pointy bars in early-type galaxies (see, e.g., Quillen et al. 1994; Ohta 1996; Buta et al. 2006).

3 The Python package galpy for Galactic Dynamics by Bovy (2015) lives at http://github.com/jobovy/galpy. 


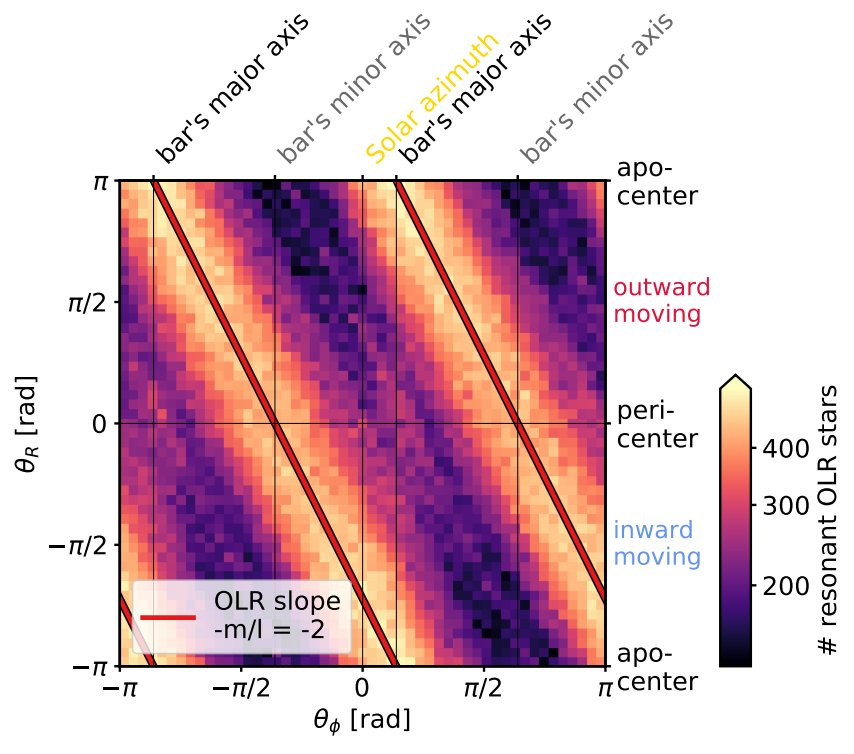

Figure 3. Angle space $\left(\theta_{\phi}, \theta_{R}\right)$ distribution of stars in the Fiducial_40 simulation that satisfy the resonance condition in Equation (20) for $l=$ $+1, m=2$, i.e. are at the bar's Outer Lindblad Resonance (OLR). The characteristic slope of $\Delta \theta_{R} / \Delta \theta_{\phi}=-2$ might help to identify the real Galactic bar's OLR in the Gaia data.

are rotating in the inertial frame in $\phi$ and oscillating in $R$. In the Fiducial_40 simulation, we can therefore determine the dominant orbital frequencies $\Omega_{\phi \text {,true }}$ and $\Omega_{R \text {,true }}$ from the maximum peak of the Fourier transform of the star's time evolution in $R(t)$ and $\cos \phi(t)$ (e.g. Binney \& Spergel 1982; Laskar 1993; Fragkoudi et al. 2019 and Figure A1) with a precision of $2 \pi /\left(T_{\text {int }}\right)=2 \pi /\left(50 T_{\text {bar }}\right)=$ $0.8 \mathrm{~km} / \mathrm{s} / \mathrm{kpc}$. We select stars in the simulation that are (not in the axisymmetric estimate but) actually in the barred system in resonance with the bar's OLR $(l=1, m=2)$ using the resonance condition

$$
\left|\Omega_{\mathrm{bar}}-\Omega_{\phi, \text { true }}-\frac{l}{m} \times \Omega_{R, \text { true }}\right|<0.8 \mathrm{~km} / \mathrm{s} / \mathrm{kpc} .
$$

Their distribution peaks around the parent orbits (Equation 13) that all follow the ( $2 \pi$-wrapped) line

$$
\begin{aligned}
\theta_{R, x_{1}(1)} & =-2\left(\theta_{\phi}-\phi_{\text {bar }}\right)+\theta_{\text {slow }, x_{1}(1)}, \\
\text { with } \theta_{\text {slow }, x_{1}(1)} & \equiv \pi
\end{aligned}
$$

(c.f. Binney 2018, eq. 39), with a slope of $-m / l=-2$ and with pericenter at the bar's minor axis. These orbits belong to the class of $x_{1}$ (1) OLR orbits aligned with the bar. Some OLR stars are found in Figure 3 on orbits that have their pericenters close to the bar's major axis; in our simulation, these exhibit low libration and belong to the anti-aligned $x_{1}(2)$ orbit class, which lives inside of the OLR ARL. Its parent orbits follow lines

$$
\begin{aligned}
\theta_{R, x_{1}(2)} & =-2\left(\theta_{\phi}-\phi_{\text {bar }}\right)+\theta_{\text {slow }, x_{1}(2)}, \\
\text { with } \theta_{\text {slow }, x_{1}(2)} & \equiv 0 .
\end{aligned}
$$

Figure 3 confirms for the OLR that-as suggested in Section 2.3 - a stellar sub-selection, which contains stars on a range of true $l: m$ resonant orbits, creates angle overdensities along stripes with the characteristic slope of $-m / l$.

\subsection{Selecting resonant stars}

No clear signal of resonances is visible in angle space when considering all stars at once in either the simulation or the Gaia data, if no pre-selection on orbits is performed. Two aspects contribute to this: (i) Stars at different bar resonances live at different $L_{z}$ and radius, but can populate overlapping regions in angle space (Figure 2) (ii) Non-resonant circulating orbits smoothly 'pollute' the whole $\left(\theta_{\phi}, \theta_{R}\right)$ plane, decreasing the contrast of the resonant signal.

The studies by Sellwood et al. (2019) and Hunt et al. (2019) show that Gaia DR2's angle space for the Solar neighbourhood $d<200$ pc exhibits strong sub-structure. ${ }^{4}$ The angle plane for all stars out to $d=3 \mathrm{kpc}$ is featureless because of the above mentioned reasons. A sub-selection on orbits is therefore crucial to isolate and reveal resonant signatures in angle space. The action plane $\left(L_{z}, J_{R}\right)$ will help with this, because — as is very well-known—resonant stars create global ridges in action space (see, e.g., Sellwood \& Binney 2002; Sellwood 2012; Binney 2018).

Using Equation (20), we select in the simulation all particles whose orbital frequencies satisfy the resonance condition for either $\mathrm{CR}$, OLR, outer 1:1, or 1:4 resonance. Figure 4 shows the fraction of these resonant stars with respect to all stars in the simulation. Overplotted are also the corresponding ARLs.

We confirm that the high- $J_{R}$ ridge to the right of the 1:2 OLR and the 1:1 ARLs (c.f. Paper I) indeed contain a high fraction of resonant stars. We call these action overdensities made up from resonant stars 'scattering ridges' (see also Paper I) to describe that on average the stellar population has been displaced towards higher $J_{R}$ at and by this resonance, as compared to the smooth initial disk. The width of the ridge depends on the libration strength around the ARL.

We will now focus on the 1:2 OLR, which gives the strongest signal, but discuss the other resonances briefly in Section 5.3 and Appendices C3-C5.

The high- $L_{z}$ edge of the OLR ridge corresponds to the outer boundary of the zone of entrapment (Binney 2018). Figure 4 illustrates this by overplotting the maximum libration amplitude at $J_{R} \sim 0.5 L_{z, 0}$. We mark this edge of the OLR ridge with a line (the 'ridge edge line', REL), for which we chose the slope $\Delta J_{R} / \Delta L_{z}=-1$ by eye. ${ }^{5}$ The majority of action ridges observed in the Gaia data by T19 have slopes steeper than the OLR ARL, but shallower than this REL. In addition, we shift the REL by $\Delta L_{z}$ with respect to the OLR ARL; in the simulation we chose $\Delta L_{z}=+0.05 L_{z, 0}$ at $J_{R}=0$. Using the REL and the ARL as boundaries for the selection will provide a stellar sub-sample with a high fraction of true OLR stars.

When creating Figure 4, we knew the true barred potential and therefore the true orbital frequencies. Neither is available for the MW, so a selection of resonant star candidates can in reality not be improved beyond selecting all stars between the OLR ARL and the REL for an assumed $\Omega_{\mathrm{bar}}$. In the left column of Figure 5,

\footnotetext{
4 The reason that the angle plane for $d<200 \mathrm{pc}$ has a lot of substructure is the following: The in-plane phase space $\left(R \sim R_{0}, \phi \sim \phi_{0}, v_{R}, v_{\phi}\right)$ is here essentially $2 \mathrm{D}$. Studying $\left(v_{R}, v_{\phi}\right)$ isolates the orbit structure therefore very cleanly. For this small volume, any 2D projection of the same phase-space, e.g. to $\left(L_{z}, J_{R}\right)$ or $\left(\theta_{\phi}, \theta_{R}\right)$, reveals the same overdensities. This can be well seen in figs. 2-3 by Hunt et al. (2019).

5 At low $J_{R}$, this choice of REL is not a good description for the real zone of entrapment and the separatrix at the OLR, as can be seen in Figure 4 and as discussed in Binney (2020a), but we found that for our practical purposes approximating it by a straight line is sufficient.
} 


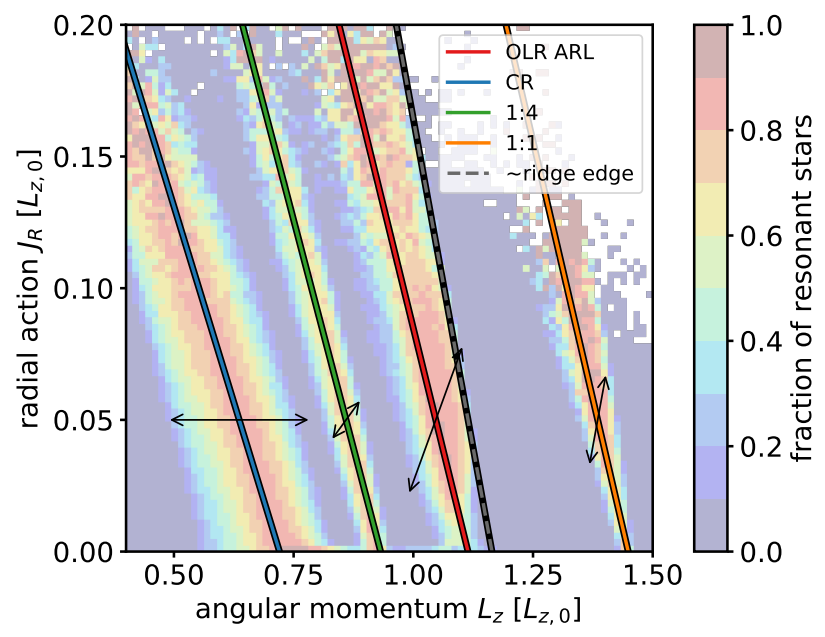

Figure 4. Fraction of resonant stars to the total number of stars in the Fiducial_40 simulation as a function of action space $\left(L_{z}, J_{R}\right)$. Resonant stars are selected using the resonance condition in Equation (20) for $l: m=$ $0: 1,1: 4,1: 2,1: 1$. Overplotted are the corresponding ARLs. The dashed line with slope $\Delta J_{R} / \Delta L_{z}=-1$ follows roughly the edge of the OLR's scattering ridge. OLR resonant stars can be found in the ridge; 1:4 resonant stars are mixed with the background for the weak $m=4$ bar component used in this work. The black arrows illustrate the direction and maximum amplitude of libration around the ARLs.

we demonstrate that this proposed selection in action space (upper left panel) works well to isolate enough stars on OLR $x_{1}$ (1) orbits to make the OLR's angle signature of slope -2 and pericenter at $\theta_{\phi, \text { peri }}=\theta_{\text {slow }} / m+\phi_{\text {bar }}$ visible (lower left panel) ${ }^{6}$

\subsection{Dealing with selection effects around the Sun}

If we had access to stars in the whole Galactic disk, $\phi \in[-\pi, \pi]$ (as in the first column of Figure 5), we could not only identify the bar's real OLR and pattern speed from the sloped stripes in angle space, but their location in $\theta_{\phi}$ would also reveal the bar's orientation with respect to the Sun.

Unfortunately, Gaia provides currently only precise data around the Sun, $\mathbf{x}_{\odot}=\left(R_{0}, \phi_{0}, z_{0}\right)=\left(8 \mathrm{kpc}, 0^{\circ}, 25 \mathrm{pc}\right)$, out to distances of roughly $d_{\max } \sim 3 \mathrm{kpc}$, even when using improved distance estimates by, e.g., Schönrich et al. (2019). As demonstrated in the second column of Figure 5, this reduces the observable $\theta_{\phi}$ range within the Galaxy to

$\left|\theta_{\phi}\right| \sim|\phi|<\arcsin \left(\frac{d_{\max }}{R_{0}}\right)$.

Near-circular orbits crossing this survey volume can be observed at any radial phase $\theta_{R}$. For orbits with high $J_{R}$, only parts of their epicycles enter the survey volume. If a star is observed close to the Sun, but its guiding center is azimuthally in front of the Sun $\left(\theta_{\phi}>0\right)$, it will currently be inward-moving $\left(\theta_{R}<0\right.$; see Figure $1)$; if a star is observed while its guiding center is behind the Sun $\left(\theta_{\phi}<0\right)$, it will be outward-moving $\left(\theta_{R}>0\right)$. These high- $J_{R}$ stars make up the $\left|\theta_{\phi}\right|>\arcsin \left(d_{\max } / R_{0}\right)$ wings in the corresponding angle distribution. How extended these wings are depends on the population properties, in particular on the radial velocity dispersion

\footnotetext{
6 If we knew the true $\Omega_{\mathrm{bar}}$ of the MW, varying the REL slope and $\Delta L_{z}$
} could be used as a constraint on the strength of the bar.
$\sigma_{R}$. If the data are complete within this survey volume, density variations along the OLR slope are still discernible in angle space.

However, the completeness of surveys depends also on the apparent brightness of the stars (e.g. Boubert \& Everall 2020 for Gaia DR2). For simplicity, we assume the completeness decreases with distance from the Sun $d$ as

completeness $(d) \propto \exp \left(-\frac{d}{1 \mathrm{kpc}}\right)+$ const.

We apply this to the data from the Fiducial_40 simulation in Figure 5, last column.

To understand how the incompleteness shapes the angle distribution, it is convenient to mark the location in the $\left(\theta_{\phi}, \theta_{R}\right)$ plane at which the average azimuth of the stars $\langle\phi\rangle \sim 0$. This line runs (in an almost axisymmetric disk, and as a function of $\theta_{R}$ ) along the peak of the stellar number distribution, as can be seen in the lower right panel of Figure 5. The ' $\langle\phi\rangle \sim 0$ ' line and the peak line agree for this completeness function by construction, because most stars are observed at the Solar azimuth $\phi=0$ (at a given $R$ ). The S-shape of the peak ' $\langle\phi\rangle \sim 0$ ' line in Figure 5 can be explained as follows: At peri- and apocenter, $\theta_{R} \in\{ \pm \pi, 0\}, \theta_{\phi}$ corresponds to the real $\phi$ of the star (c.f. McMillan 2011, fig. 1). Consequently, at these $\theta_{R} \mathrm{~s}$, the maximum number of stars is observed at $\phi=\theta_{\phi}=0$. The many stars that are currently at $\phi=0$, but have $\theta_{R}$ around $+\pi / 2$ or $-\pi / 2$, have guiding centers further away from the Sun, at $\theta_{\phi}<0$ or $\theta_{\phi}>0$, respectively. Here $\theta_{\phi} \neq \phi$, and the exact location of the density peak depends on the radial velocity dispersion. By going away from this peak line in $\theta_{\phi}$, the number density decreases (and $|\langle\phi\rangle|$ increases) smoothly towards the wings of the distribution.

A pedagogic explanation of the selection effects in angle space can also be found in the appendix of Hahn et al. (2011).

For this completeness function, the physical angle signature of the OLR is hidden by selection effects in the lower right panel of Figure 5. If we knew the real form of Gaia's incompleteness function (like Equation (26) for the Fiducial_40 simulation), it would be possible to perform full-likelihood forward-modeling of the angle distribution to uncover the OLR signature (c.f. also McMillan 2011).

Here, we present a simpler strategy to make the OLR's angle signature visible that does not require precise knowledge of the selection function. We make use of the fact that an incompleteness function like that presented in Equation (26) affects outward- and inward-moving stars in equal measures, leading to the symmetric $S$-shaped envelope in angle space. We mirror the angle distribution twice-horizontally at $\theta_{\phi}=0$ and vertically ${ }^{7}$ at $\theta_{R}=0$ - and calculate the relative number difference between the original and the mirrored angle distribution. By doing so, we effectively remove the incompleteness decrease at $|\phi|>0$. Physically, this corresponds to comparing the number of outward moving stars with the same guiding center location $\theta_{\phi}$,

$N_{i}^{+} \equiv N_{i}\left(\theta_{\phi, i}, \theta_{R, i}\right)$

with the number of inward-moving stars that have guiding center locations on the opposite $\phi$-side with respect to the Sun, i.e.

$N_{i}^{-} \equiv N_{i}\left(-\theta_{\phi, i},-\theta_{R, i}\right)$.

As both bins should be affected in the same way by selection effects, we scale the difference by the total number of stars in these two bins.

\footnotetext{
7 The results do not change if the mirroring in $\theta_{R}$ is performed at pericenter or at apocenter.
} 

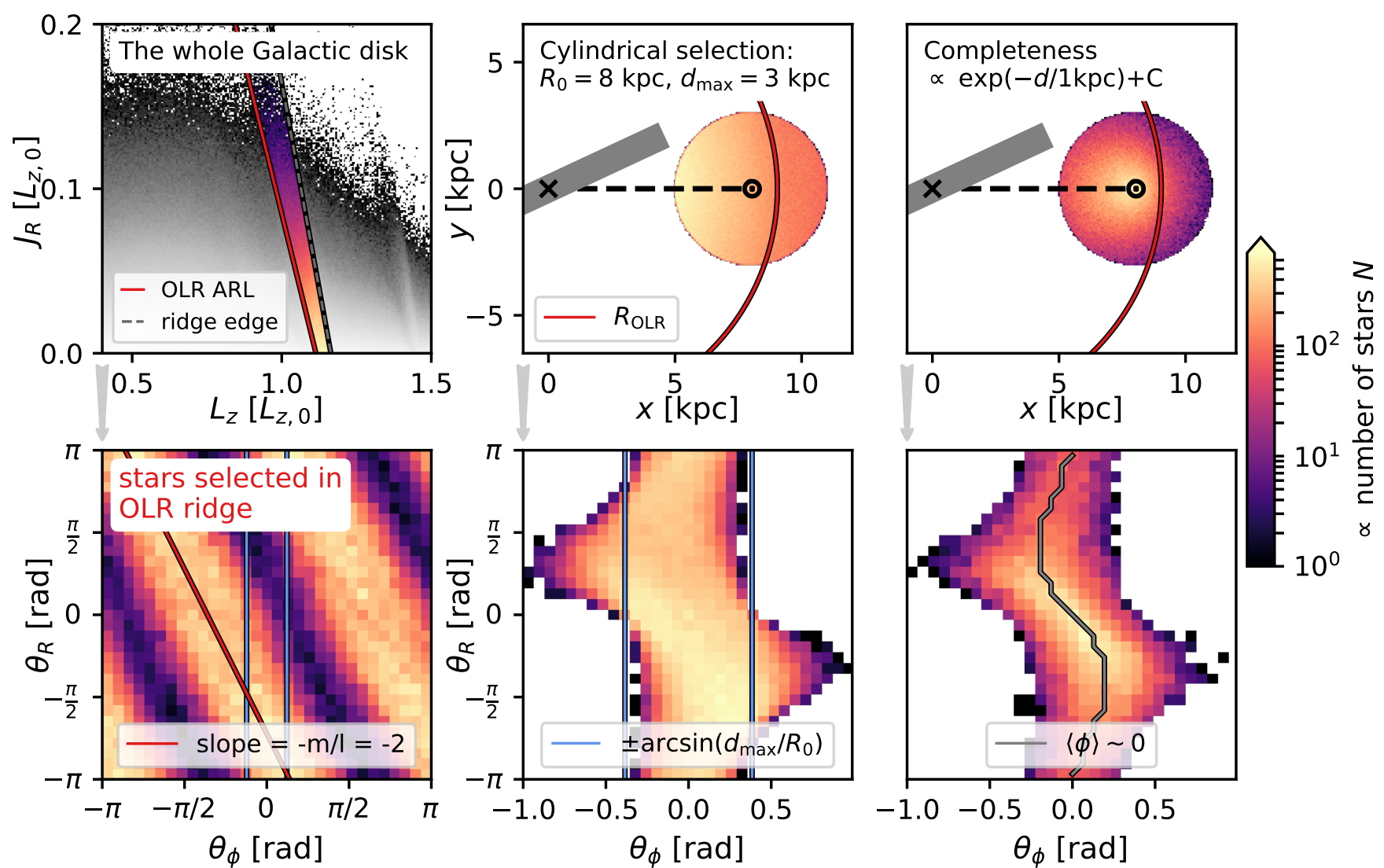

Figure 5. Illustration of survey selection effects in angle space (in the Fiducial_40 simulation). 1st column: The characteristic OLR slope of -2 in angle space is still clear when selecting stars directly from the scattering ridge in action space for the whole disk. 2nd column: Restricting the data to a cylinder around the Sun reduces the $\theta_{\phi}$ range substantially. 3rd column: An additional completeness function is imposed that decreases with distance. The stellar density peaks now at the Solar azimuth $\phi=0$, corresponding to an S-shaped curve in angle space (see text for details). This selection effect hides the OLR signature.
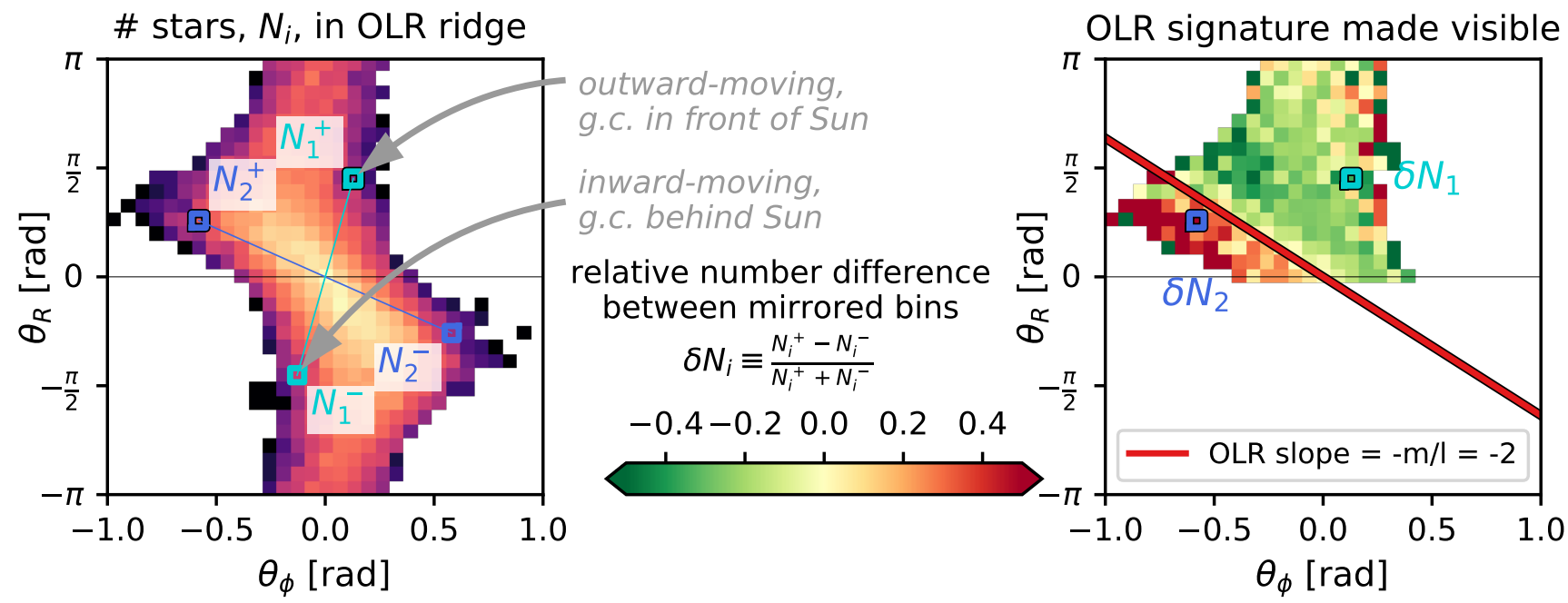

Figure 6. Dealing with selection effects: Completeness decreasing with distance from the Sun (in the Fiducial_40 simulation). Left: Stars from the OLR ridge in angle space (same as lower right panel in Figure 5). We compare the number of stars at $N_{i}^{+} \equiv\left(\theta_{\phi}, \theta_{R}\right)$ with those at $N_{i}^{-} \equiv\left(-\theta_{\phi},-\theta_{R}\right)$. Right: colour-coding angle space $\left(\theta_{R}>0\right)$ by the relative stellar number difference makes the OLR slope visible. 

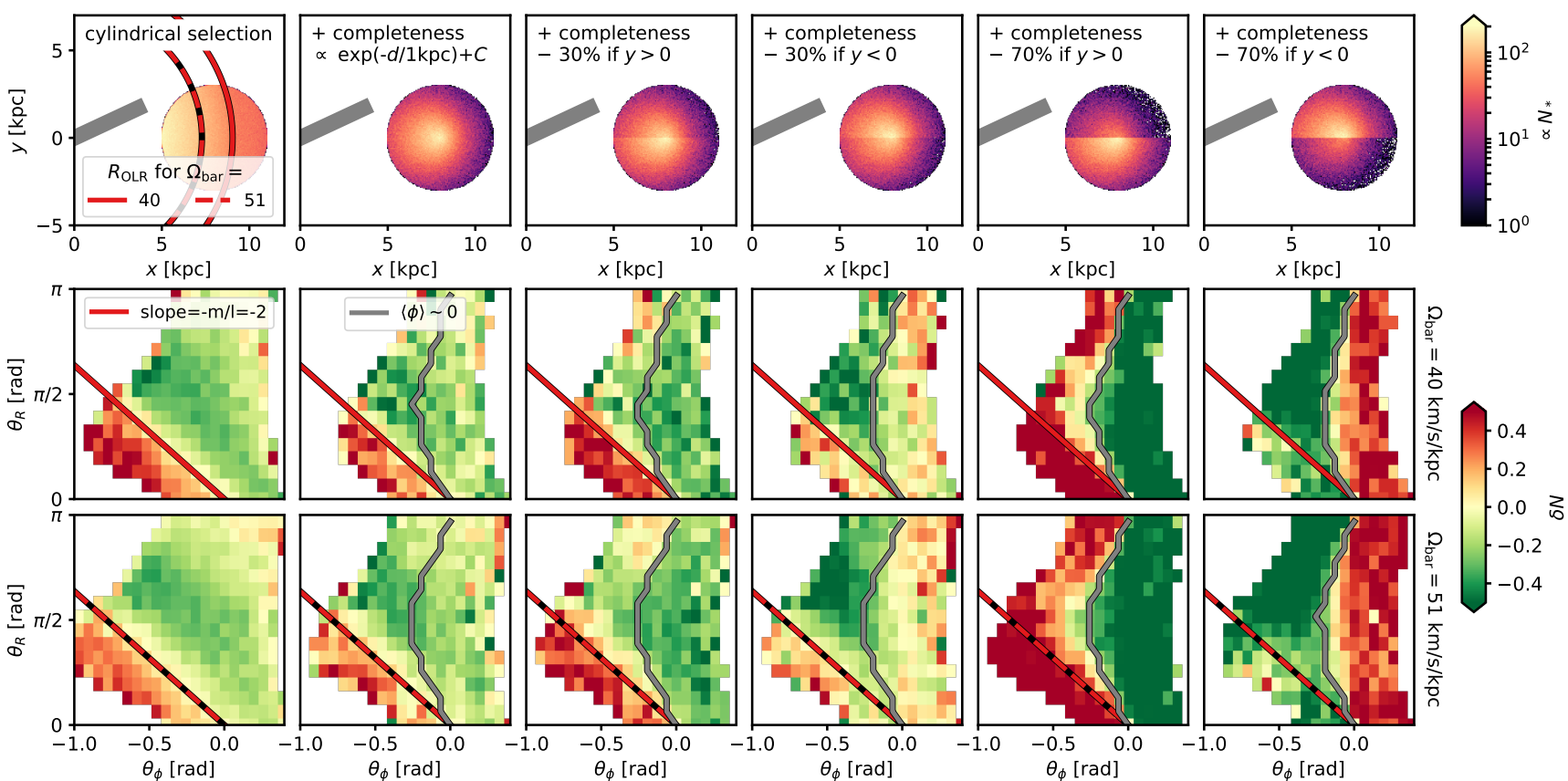

Figure 7. Dealing with selection effects: Completeness variation with Galactic longitude. For two simulations with different bar pattern speeds (Fiducial_40 and Fiducial_51), it is shown how the clean OLR signature in relative star numbers $\delta N$ ( 1 st and 2 nd column) is still visible for completeness differences of up to $30 \%$ ( $3 \mathrm{rd}$ and 4 th column), but is overtaken by selection effects for completeness differences of $70 \%$ (5th and 6 th column). In the latter case, the pattern in $\delta N$ follows the shape of the selection-function-affected stellar number distribution (denoted by the grey line along which $\langle\phi\rangle \sim 0$ ) rather than the OLR slope (red line).

We illustrate this strategy in Figure 6. The relative residuals,

$\delta N_{i} \equiv \frac{N_{i}^{+}-N_{i}^{-}}{N_{i}^{+}+N_{i}^{-}}$,

reveal now the OLR signature visually and clearly by transitioning through zero along the line

$\theta_{R}=-2 \theta_{\phi}$,

which we call OAS ("OLR angle slope" line). The OAS line goes through $\left(\theta_{\phi}, \theta_{R}\right)=(0,0)$ by construction, and the region $\theta_{R}<0$ does not contain additional information because of the mirroring. For $\theta_{R}>0$, the distribution below the OAS line has $\delta N>0$ ('red' = dominated by outward-moving stars), and above $\delta N<0$ ('green' = dominated by inward-moving stars). This tells us that we are looking at $x_{1}(1)$ orbits. For $x_{1}(2)$ orbits, it would be the other way round.

The width of the 'green' stripe above the OAS line in principle still contains some information about the bar orientation angle and the width of the unmirrored stripe due to the libration strength of the OLR orbits, but requires careful modeling. In this work, we will just focus on investigating the pattern in $\delta N$ around the OAS line.

\subsection{Dealing with selection effects due to asymmetric sky coverage}

In the previous section, we showed how the OLR's angle signature can be made visible in the case of a symmetric incompleteness function with respect to the Sun. In particular, the symmetry in Galactic longitude $- \pm l$ with respect to the Galactic center-is crucial for our method to work: Stars with the same $\left|\theta_{R}\right|,\left|\theta_{\phi}\right|$, and $\left(L_{z}, J_{R}\right)$ live at the same $\pm \phi$ and thus $\pm l$. The completeness symmetry in $l$ is therefore more important for obtaining a physically meaningful $\delta N$ (Equation 29) than the absolute value of the completeness.

The assumption of longitude symmetry might, however, be too simplified for real MW data. Boubert \& Everall (2020) investigated Gaia DR2's completeness by modeling the survey's sky coverage using Gaia's scanning law. The latter is not perfectly symmetric with respect to the Galactic center. Rybizki et al. (2021) constructed an empirical completeness function of the Gaia DR2 RVS sub-sample with respect to the full DR2 catalogue. Within the magnitude range

$5<G_{\mathrm{RVS}} / \operatorname{mag}<12$

and colour range

$0.35<\left(G-G_{\mathrm{RP}}\right) / \mathrm{mag}<1.25$

this internal completeness is $80 \%$ or better (see fig. 3 in Rybizki et al. 2021). The internal completeness varies with position on the sky. By comparing the completeness function ${ }^{8}$ by Rybizki et al. (2021) at locations $+l$ vs. $-l$, the differences are mostly lower than $15 \%$ across the sky. An exception are the regions $|l| \in\left[10^{\circ}, 30^{\circ}\right]$, $b \in\left[-20^{\circ},+20^{\circ}\right]$, where the completeness differences can be up to $\sim 30 \%$.

Rybizki et al. (2021) argue that the internal completeness of Gaia RVS can be considered as an external completeness as well.

\footnotetext{
8 A Python tutorial for the Gaia DR2 RVS selection function by Rybizki et al. (2021) can be found at https://github.com/jan-rybizki/ gdr2_completeness/blob/master/tutorials/ $\% 5 \mathrm{~B} 5 \% 5 \mathrm{D} \% 20 \mathrm{RVS}$ selection_function.ipynb.
} 
Rybizki \& Drimmel (2018) have investigated ${ }^{9}$ that Gaia DR2 itself is almost complete with respect to 2MASS (Skrutskie et al. 2006) in the magnitude range $8<G / \mathrm{mag}<12$. Completeness differences of up to $\sim 30 \%$ can therefore be considered as a realistic value also for the Gaia RVS sample with respect to the true stellar distribution.

To investigate if such completeness differences in $\pm l$ can hide the physical OLR signature in angle space, we remove $0 \%, 30 \%$, or $70 \%$ of the test particles at Galactic coordinates $y>0$ or $y<0$ from both the Fiducial_40 and Fiducial_51 simulations. Figure 7 compares the corresponding $\delta N$ distributions with the OAS line and the ' $\langle\phi\rangle \sim 0$ ' line in angle space.

We find that for $70 \%$ completeness differences the 'red/green' pattern follows the ' $\langle\phi\rangle \sim 0$ ' line because of the imposed completeness break at $\phi=0$. We conclude from this that if the $\delta N$ distribution follows closely the ' $\langle\phi\rangle \sim 0$ ' line, there is a danger that what we are seeing is not a physical signature, but rather a selection effect.

In the case of $30 \%$ completeness differences, the pattern in $\delta N$ still follows cleanly the OAS line, just as in the ideal case of $0 \%$ completeness difference. We conclude therefore that the completeness variation across $\pm l$ in Gaia DR2 RVS are not strong enough to hide the physical resonance signatures in angle space.

\section{RESULTS FROM THE GAIA DATA}

\subsection{Gaia DR2 RVS data}

We use the Gaia DR2 (Gaia Collaboration et al. 2018a) sub-sample that has, in addition to on-sky positions and proper motions, also radial velocity measurements from Gaia's Radial Velocity Spectrometer (RVS, Katz et al. 2019). We clean this sample by applying the cuts in brightness and colour (Equations 31-32) proposed by Rybizki et al. (2021). For the distances to the stars, we use the Bayesian estimates by Schönrich et al. (2019) and cut at $d \leq 3 \mathrm{kpc}$ to ensure good accuracy and precision.

We transform to Galactocentric coordinates using the Solar peculiar motion measurements by Schönrich et al. (2010), $R_{0}=8 \mathrm{kpc}$ and $v_{\text {circ }}\left(R_{0}\right)=220 \mathrm{~km} / \mathrm{s}$ in the gravitational MWPotential2014 model by Bovy (2015), as well as $z_{0}=25$ pc (Jurić et al. 2008). Axisymmetric actions, angles, and frequencies are estimated in the MWPotential2014 using the Stäckel fudge algorithm (Binney 2012), just as for the test particles in the simulation, and as done for the Gaia data in T19 and Paper I. A further cut is imposed to focus on orbits with small vertical excursions close to the Galactic plane $\left(J_{z}<0.01 L_{z, 0}\right)$.

We present Gaia's full 4D action-angle space in Appendix B in Figure B1.

As we will show in Appendix D, the details of the assumed gravitational MW potential do not matter for the subsequent analysis. In particular, we have confirmed the qualitative results that we will present in the next sections also for the MW model by Eilers et al. (2019).

Using Gaia EDR3 (Gaia Collaboration et al. 2021) rather than DR2 would also not affect our results. Proper motion uncertainties $\delta \mu$ are by a factor 2-3 smaller in EDR3 than in DR2. For the previously already very precisely measured DR2 proper motions

9 The tutorial by Rybizki \& Drimmel (2018) showing the completeness of Gaia DR2 with respect to 2MASS, can be found at https://github. com/jan-rybizki/gdr2_completeness/blob/master/tutorials/ $\% 5 \mathrm{~B} 2 \% 5 \mathrm{DC}$ ompleteness $\% 20$ tutorial_gdr2_light.ipynb.

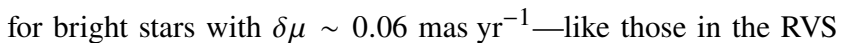
sample - this improvement does not have a significant impact. Gaia parallaxes have not been used in this work.

\subsection{Candidates for the MW bar's OLR in angle space}

Based on the resonant action-angle-signature presented in Section 3, we will now search in the Gaia data for the bar's true OLR. For this, we adopt the following independent approach, which is also illustrated in Figure B1 in Appendix B.

Step 1: Picking a bar pattern speed $\Omega_{\text {bar }}$, and finding the location of the corresponding OLR ARL in action space $\left(L_{z}, J_{R}\right)$.

Step 2: Assuming that a large fraction of true OLR stars is found to the right of the ARL, and therefore selecting stars between the ARL and an assumed REL with slope -1 and shifted by $\Delta L_{z}=+0.01 L_{z, 0}$ with respect to the ARL at $J_{R}=0 .{ }^{10}$

Step 3: Comparing the $\delta N\left(\theta_{\phi}, \theta_{R}\right)$ distribution of this stellar subselection with the model prediction for true OLR $x_{1}(1)$ orbits in the simulation (Figure 6 and lower left in Figure B1) along the OAS line of slope -2 to identify if this could be a candidate for the real OLR - and therefore for the bar pattern speed.

Step 4: Comparing $\delta N\left(\theta_{\phi}, \theta_{R}\right)$ also to the ' $\langle\phi\rangle \sim 0$ ' line to check if selection effects might 'fake' the OLR signature (see Figure 7).

Step 5: Repeating Steps 1-4 for varying $\Omega_{\mathrm{bar}}$. By doing so, we effectively scan with the OLR ARL across the action plane (upper left panel in Figure B1) and study the corresponding angle planes (right panels in Figure B1).

The structures in the Gaia data are complex-also in angle space. A meaningful quantitative criterion to decide which angle plane is the one and only true bar OLR is therefore beyond our current ability to understand and model the data. We restrict ourselves therefore to a qualitative (and therefore disputable) by-eye identification of possible candidates. The reader is encouraged to read-off in Figure B1 the $\Omega_{\text {bar }}$ for which they themselves think the data look closest to the model prediction.

For the casual reader, we recommend to jump directly to Figure 8 , where we present four independently identified OLR candidates that we think look convincing: $\Omega_{\text {bar }} \in\{1.2,1.4,1.6,1.74\} \Omega_{0}$.

- $\Omega_{\mathrm{bar}}=1.2 \Omega_{0}=33 \mathrm{~km} / \mathrm{s} / \mathrm{kpc}$ associates the Hat ( $\mathrm{I}$, yellow) action ridge with the OLR and is interestingly very similar to slow bar models deduced from the local velocities in the literature (c.f., e.g., Monari et al. 2019a; Binney 2020b; Paper I; Kawata et al. 2021). Unfortunately, the angle data are noisy and restricted to close-to-pericenter stars and the ' $\langle\phi\rangle \sim 0$ ' line overlaps with the OAS line, suggesting that we might see selection effects here rather than a physical signature.

- $\Omega_{\text {bar }}=1.4 \Omega_{0}=38.5 \mathrm{~km} / \mathrm{s} / \mathrm{kpc}$ suggests that the Sirius (G1, orange) ridge is close to the OLR (c.f. Kawata et al. 2021). This pattern speed is in our opinion the strongest candidate in angle space. Surprisingly, this measurement is consistent with direct measurements of the bar pattern speed in the Galactic center (c.f.

10 For the test particle simulations we have used $\Delta L_{z}=0.05 L_{z, 0}$ as compared to $\Delta L_{z}=0.01 L_{z, 0}$ in the Gaia data. As can be seen in Figure 4, also a smaller action area to the right of the ARL would contain many OLR stars. The Gaia data are so plentiful that also this smaller area provides excellent number statistics. 


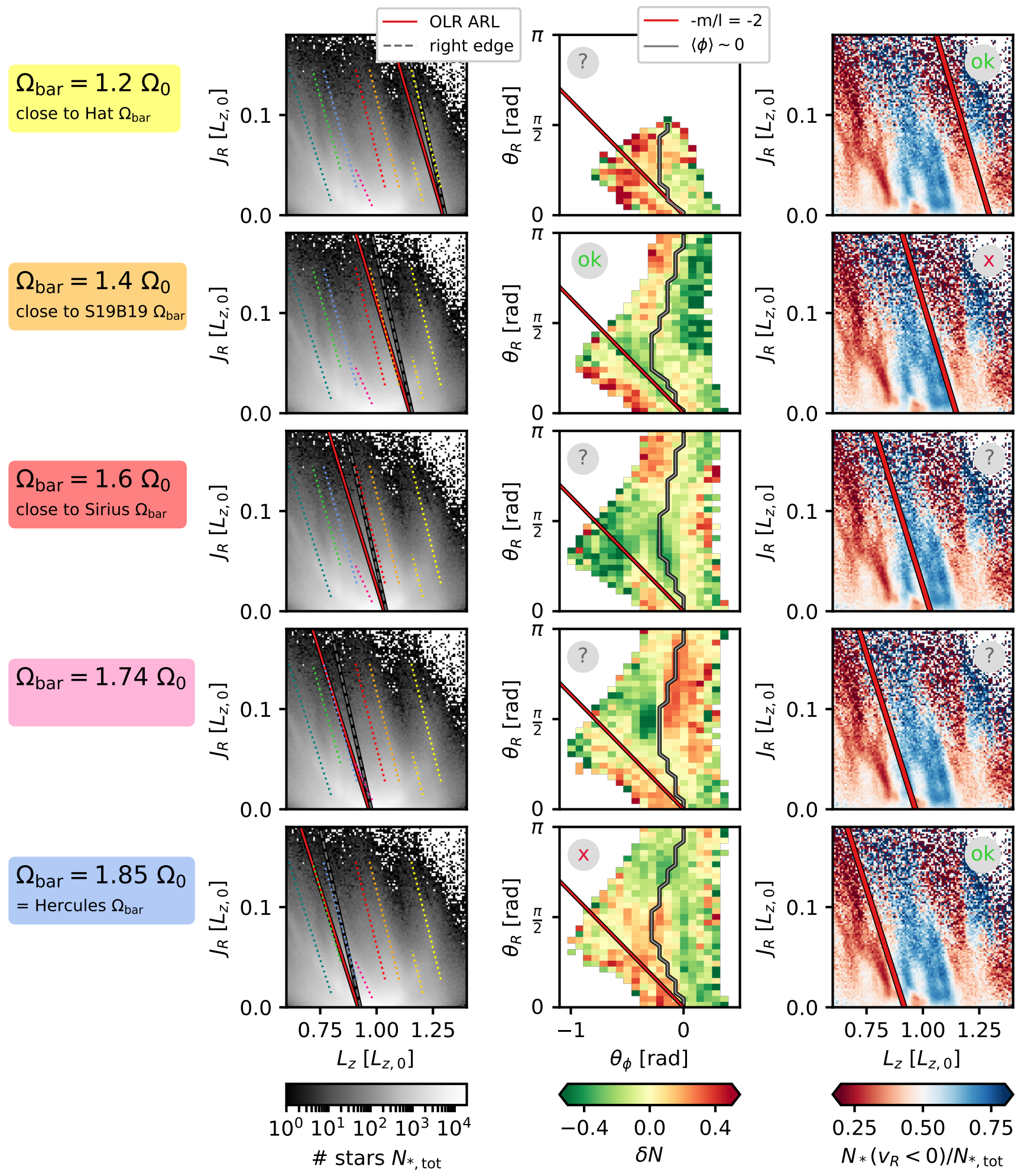

Figure 8. OLR candidates in the Gaia DR2 action-angle data. Middle: Four possible pattern speed candidates were identified from Gaia's full angle space in Figure B1 and are now presented here: $\Omega_{\text {bar }} \in\{1.2,1.4,1.6,1.74\} \Omega_{0}$. The corresponding angle planes show that the red OAS separates a 'red' from a 'green' stripe in $\delta N$, as expected for the OLR. Interestingly, the first three candidates agree with favourites in the literature. Left: In the full action space, the red OLR ARL and grey dashed line mark the respective sub-selection of stars shown in angle space. The dotted lines denote the moving group ridges following T19, e.g., the Hat (I, yellow), Sirius (G1, orange), and Hercules/Horn (D1, blue). Right: In addition, we show the location of the OLR ARL on top of the $v_{R}$-asymmetry in $\left(L_{z}, J_{R}\right)$. A 'red/blue' signature (c.f. Paper I and references therein) would be expected around the true OLR. Because of selection effects in angle space at the Hat, or not fully understood mechanisms causing Sirius, no clear preference for either the slow Hat or slightly faster slow S19B19 $\Omega_{\mathrm{bar}}$ can be reached. The fast Hercules bar pattern speed, shown for comparison at the bottom, can, however, be ruled out by the angle distribution. 
Portail et al. 2017; Sanders et al. 2019; Clarke et al. 2019; Bovy et al. 2019; Pérez-Villegas et al. 2017).

- $\Omega_{\text {bar }}=1.6 \Omega_{0}=44 \mathrm{~km} / \mathrm{s} / \mathrm{kpc}$ - with the OLR located between the Hyades/Horn (E1, pink) and the Sirius (F1, red) ridges - is a weak candidate because of the large inward-moving ('green') blob at $\theta_{R} \sim \pi / 4$. But we point out that at least at low $\theta_{R}$ the $\delta N$ pattern follows exactly the OAS, while the ' $\langle\phi\rangle \sim 0$ ' line has a very different slope, suggesting that this signature is physical. This pattern speed is close to suggestions by Kalnajs (1991), Hunt et al. (2019), Paper I, and Kawata et al. (2021) from the local action space, and to measurements from the Galactic center, $41 \pm 3 \mathrm{~km} / \mathrm{s} / \mathrm{kpc}$ (Sanders et al. 2019; Bovy et al. 2019).

- $\Omega_{\text {bar }}=1.74 \Omega_{0}=47.9 \mathrm{~km} / \mathrm{s} / \mathrm{kpc}$ aligns the OLR ARL with the 'Pleiades', the 'Hyades' and the 'Horn' (the Hyades/Horn (D1, pink) ridge in action space) and the signature along the OAS in angle space is distinct from possible selection effects.

Even the last pattern speed is slower than the classical and wellconstrained fast bar pattern speed of $\Omega_{\mathrm{bar}}=1.85 \Omega_{0}=51 \mathrm{~km} / \mathrm{s} / \mathrm{kpc}$ in the literature (Dehnen 2000; Antoja et al. 2014) that associates the 'Hercules/Horn' bimodality with the OLR (see also, e.g., Fragkoudi et al. 2019; this corresponds to the Hercules (C1, green) and Hercules/Horn (D1, blue) ridges in action space). For comparison, we show in the bottom row of Figure 8 also the angle space for this Hercules pattern speed. In this region of phase-space, the ' $\langle\phi\rangle \sim 0$ ' line does not follow the observed 'red/green' transition in $\delta N$; based on Section 3.4, selection effects are therefore not responsible for the observed 'red/green' pattern. The latter might therefore be a true dynamical signature. The 'red/green' transition does, however, not agree with the OAS. We can therefore state that there are no angle signatures consistent with $x_{1}$ (1) OLR orbits at the location of the classical fast bar's OLR.

This result of ruling out 'Hercules' as an OLR candidate is in agreement with recent work that also looked at the extended phasespace structure of 'Hercules' in Gaia DR2 RVS. Binney (2020b) found-using torus-based modeling - that the velocity structure of 'Hercules', at different locations within $1 \mathrm{kpc}$ from the Sun, is not consistent with trapping at the OLR. Monari et al. (2019c) pointed out that the slope of the 'Hercules' $\left\langle v_{R}\right\rangle$ feature in the $\left(L_{z}, \phi\right)$ plane, disagrees with the OLR prediction. Both these works prefer the CR as origin of 'Hercules'.

\section{DISCUSSION}

\subsection{Revisiting the OLR's orbit orientation flip in action space}

Several strategies have been employed in the literature to identify the bar's OLR in the local stellar phase-space (e.g., Dehnen 2000; Mühlbauer \& Dehnen 2003; Chakrabarty 2007; Minchev et al. 2010; Antoja et al. 2014; Monari et al. 2017a; Tian et al. 2017; Monari et al. 2019b; Khoperskov et al. 2020; Hinkel et al. 2020a; Paper I). Many use the well-known fact that the orientation of disk orbits with respect to the bar flip at the OLR from $x_{1}(2)$ orbits inside of the OLR to $x_{1}(1)$ orbits outside of the OLR (e.g., Contopoulos \& Grosbol 1989; Kalnajs 1991; Sellwood \& Wilkinson 1993; Dehnen 2000; Fux 2001b; Binney \& Tremaine 2008; Fragkoudi et al. 2019). At the Solar azimuth $\sim 25^{\circ}$ behind the bar, this translates to a flip from outward- to inward-moving stars.

In Paper I, we used this orbit orientation flip to point out a simple strategy to directly read off all possible OLR candidates and associated bar pattern speeds from the $\left(L_{z}, J_{R}\right)$ plane colour- coded by the radial velocity. Specifically, the 'fraction of inward moving stars', $N_{*}\left(v_{R}<0\right) / N_{*}$,tot, tells us which features in action space are predominantly outward-moving (colour-coded 'red') or inward-moving (colour-coded 'blue'). This is a proxy of the average radial phase, $\left\langle\theta_{R}\right\rangle$, rather than the average radial velocity, $\left\langle v_{R}\right\rangle$. We found that in simulations and for survey volumes out to several kpc, the OLR's $v_{R}$-signature can be studied very well in axisymmetric action space, where the ARL neatly separates the 'red' from the 'blue' feature.

In Paper I, we identified 'red/blue' features around OLR ARLs in Gaia DR2 RVS for the following pattern speeds:

- $\Omega_{\text {bar }}=33 \mathrm{~km} / \mathrm{s} / \mathrm{kpc}=1.2 \Omega_{0}$, with the Hat as the inward-moving OLR ridge, and the gap between 'Hat' and 'Sirius' as the outwardmoving part of the signature (c.f. the slow bar in, e.g., Monari et al. 2019a; Hunt et al. 2019),

- $\Omega_{\mathrm{bar}}=45 \mathrm{~km} / \mathrm{s} / \mathrm{kpc}=1.63 \Omega_{0}$, with the outward-moving Sirius as the OLR ridge, and the 'Hyades' as the inward-moving feature (c.f. Kalnajs 1991),

- $\Omega_{\mathrm{bar}}=51 \mathrm{~km} / \mathrm{s} / \mathrm{kpc}=1.85 \Omega_{0}$, with Hercules as the inwardmoving feature, and the 'Horn' as the OLR ridge, (c.f. the fast bar in, e.g., Dehnen 2000; Antoja et al. 2014).

We refer the reader to Paper I, where we have discussed these candidates and their previous appearances in the literature in depth.

\subsection{Comparing the action- and angle-based methods for the OLR}

There is more than one feature in the local kinematics that looks like the bar OLR's velocity flip. But only one can be the true OLR (if at all visible) and the others will be related to other bar resonances (e.g., Monari et al. 2019a; Hunt \& Bovy 2018; Asano et al. 2020) and spiral arms (De Simone et al. 2004; Chakrabarty 2007; Quillen et al. 2018; Michtchenko et al. 2018; Hunt et al. 2019; Khoperskov et al. 2020; Hunt et al. 2020). Our angle-based method provides additional constraining evidence.

In Figure 8, we compile for all bar pattern speed candidates the two different OLR diagnostics: (i) the 'red/blue' feature in actionspace around the OLR ARL from Paper I (summarized in Section 5.1 ), and (ii) the 'slope of -2 ' feature in angle space from this work (see Section 4.2).

Interestingly, pattern speed candidates around $1.2 \Omega_{0}$ and $\sim$ $1.6 \Omega_{0}$ were independently suggested by both strategies. While for $1.2 \Omega_{0}$, the OLR ARL falls right between a 'red/blue' feature, this is not the case for our most promising candidate from angle space, $1.4 \Omega_{0}$. For $1.4 \Omega_{0}$, the ARL falls on top of the 'blue', inward-moving Sirius (G2, orange) ridge, with a prominent outward-moving underdensity at higher rather than smaller $L_{z}$ (c.f. Paper I, §6.3.4). For $1.6 \Omega_{0}$ or $1.74 \Omega_{0}$, the ARL separates 'red/blue' features only at small or high $J_{R}$, respectively. The best candidate from action and velocity space, the Hercules pattern speed $1.85 \Omega_{0}$, was strongly disfavoured in Section 4.2 due to not exhibiting the expected OLR pattern in angle space.

The different outcomes of the two methods for a given OLR candidate can reveal additional information about the true dynamical nature of the respective feature. It is therefore important to point out that these two diagnostics differ from each other as follows:

- The 'action- $v_{R}$-ARL' method requires both $x_{1}(1)$ and $x_{1}(2)$ orbits, but checks only for the associated flip in $v_{R}$.

- The 'angle- $\delta N$ ' method uses only the stars on (possible) $x_{1}(1)$ 

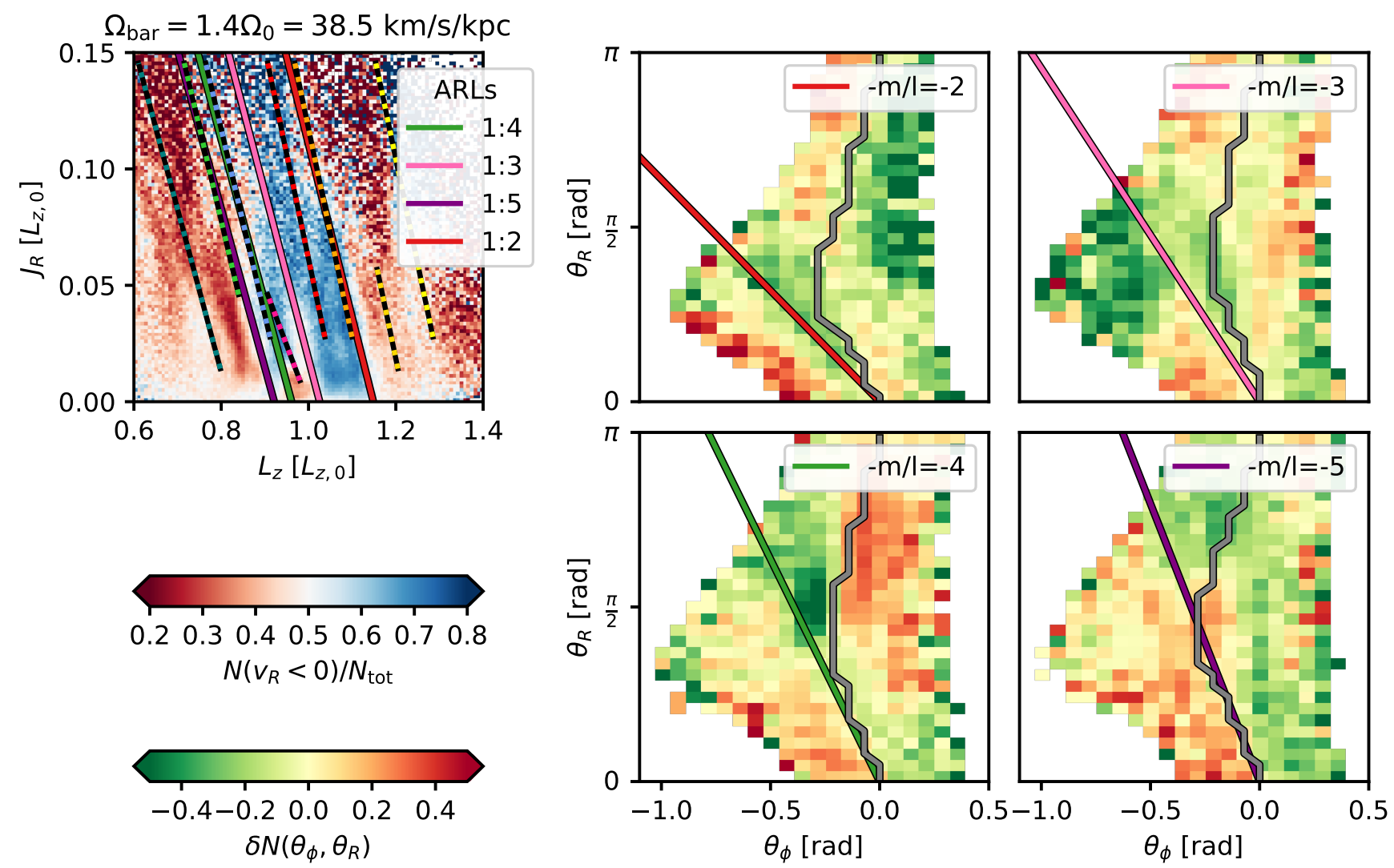

Figure 9. Angle space for stars in Gaia DR2 at higher order resonances $(l=+1, m \in\{3,4,5\})$ for the pattern speed candidate of $\Omega_{\mathrm{bar}}=1.4 \Omega_{0}=38.5 \mathrm{~km} / \mathrm{s} / \mathrm{kpc}$ that exhibits an especially convincing OLR signature $(m=2)$. Interestingly, $\Delta \theta_{R} / \Delta \theta_{\phi}=-m / l$ signatures are observed in these panels. For $m>2$, we expect these slopes resulting directly from the orbit shapes. Even though we could not reproduce these observations with our idealised simulated disk populations, the coincidence in the data is still noteworthy. The upper left panel shows the location of the ARLs (solid lines) with respect to the action ridges (dotted lines). The 1:5 and 1:4 ARLs align with sub-ridges in 'Hercules', Hercules (C1, green) and Hercules/Horn (D1, blue), respectively (c.f. T19).

orbits, but explicitly tests if the angle distribution is consistent with the expected shape of these orbits.

What mechanisms cause features that look like an OLR diagnostic in one method but not in the other? Coming up with alternative explanations is beyond the scope of this work. Considering the complex interplay of perturbers in the MW, dynamical studies of self-consistent and cosmological simulations might be required.

What is possible with our test particle simulation, however, is to bring together our action- and angle-based methods and derive two further idealized diagnostics for the bar OLR in angle space. In Appendix $\mathrm{C} 1$, we search for the orbit orientation flip in angle space, finding that we cannot find signatures of anti-aligned $x_{1}(2)$ orbits in the Gaia data. In Appendix C2, we discuss the strength of the OLR signature in angle space, which lets us rule out $\Omega_{\mathrm{bar}}=1.74 \Omega_{0}=$ $47.9 \mathrm{~km} / \mathrm{s} / \mathrm{kpc}$ from Section 4.2 as a candidate for the bar's pattern speed.

\subsection{Higher-order resonances: A curious coincidence in the Gaia data for $\Omega_{\mathrm{bar}}=1.4 \Omega_{0}=38.5 \mathrm{~km} / \mathrm{s} / \mathbf{k p c}$}

In this work, we focused on identifying OLR $x_{1}(1)$ orbits, as those left the strongest imprints in our test particle simulations. We have also searched for $\Delta \theta_{R} / \Delta \theta_{\phi}=-m / l$ signatures of other bar resonances in the Gaia angle data-but with less success. We provide some details in Appendices $\mathrm{C} 3$ for the 1:1 resonance, in $\mathrm{C} 4$ for $\mathrm{CR}$, and in $\mathrm{C} 5$ for the 1:4 resonance.

Figure 9, however, shows an interesting observation we made in the angle data for the MWPotential2014. We selected stars in the Gaia DR2 RVS action data next to the 1:4, 1:3, and 1:5 ARLs for our best $\Omega_{\text {bar }}$ candidate in Figure 8, namely $1.4 \Omega_{0}=38.5 \mathrm{~km} / \mathrm{s} / \mathrm{kpc}$. In case of this 1:4 resonance-for which the ARL aligns with the Hercules/Horn (D1, blue) ridge and which contains also stars from the Hyades/Horn (E1, pink) ridge-we observe a 'red/green' transition in angle space along a slope of -4 . For the 1:3 resonance-which falls between the Hyades/Horn (E1, pink) and the Sirius (F1, red) ridges-a transition line with a -3 slope shows up for small $\theta_{R}$. For the 1:5 resonance-which aligns its ARL with the Hercules ( 1 , green) ridge and contains also stars from the Hercules (C2, blue) ridge-a transition line with slope -5 at small and larger $\theta_{R}$ is observed.

Based on our simulations, the 'red/green' transition at this 1:4 resonance would suggest that the MW has a pointy bar with extremely strong $m=4$ component (see Appendix C5), which is not realistic. We can therefore not explain this observation on the basis of our simple simulations. Selection effects might also play a role, especially at the 1:4 and 1:5 resonance. Additional substructure is also present at $\theta_{R} \gtrsim \pi / 4$ for the $1: 3$ resonance, and around $\theta_{R} \sim \pi / 2$ for the $1: 5$ resonance.

Partly responsible for this observed coincidence is also the 
choice of potential model. A model with a different rotation curve would not necessarily have the best OLR candidate and these higherorder resonances at the same pattern speed (see Appendix D).

Monari et al. (2019b) identified candidates for higher-order bar resonances in action-velocity space for a similar bar pattern speed. Because of the differences in the assumed potential model, their 1:4 resonance was related to Sirius, the 1:3 resonance to the small gold (H) ridge in the Gaia data, and the Horn/Hyades to the 1:6 resonance. The assignment of ridges to resonances by Monari et al. (2019b) therefore clearly differs from the one suggested here.

Interestingly, Asano et al. (2020) found higher-order bar resonance features in the self-consistent MW simulation by Fujii et al. (2019) that might resemble the velocity structure of the 'Hercules' sub-arches. Their 'Hercules 2', 'Hercules 1', and 'Horn' arches would in this case be associated with 1:5, 1:4, and 1:3 bar resonances. Their 'Hercules 2' and 'Hercules 1' correspond to our Hercules (C1, green) and Hercules/Horn (D1, blue) ridges. The conclusion by Asano et al. (2020), even though based on a completely different method, is therefore consistent with our findings.

Kawata et al. (2021) argue based on the action ridges in Gaia EDR3 that both these proposals for higher-order resonances are plausible, also seeing indications for additional $-1: 4,+3: 4$, and $+1: 1$ bar resonances.

\subsection{Discussion of previous work on resonant angle signatures at the 'Hyades'}

In the past, the observational restriction to stars within $\sim 200 \mathrm{pc}$ prohibited a more extensive exploitation of the phase-angles. Stars with $L_{z} \ll L_{z, 0}$ were only observed close to apocenter, those with $L_{z} \gg L_{z, 0}$ close to pericenter. Only for orbits with $L_{z} \sim L_{z, 0}$ could a large range of radial phases be probed. The 'Hyades' moving group, $(U, V) \sim(-33,-16) \mathrm{km} / \mathrm{s}$, and the associated ridge in action space around $L_{z} \sim 0.95 L_{z, 0}$, was therefore the best candidate for an action-angle exploration.

Sellwood (2010) was the first to use angle space as a diagnostic for resonances. When investigating action space, he found that the ridge of the 'Hyades' stream lies along a line on which the resonance condition (6) is satisfied, i.e. along an ARL in our nomenclature. ARLs for different $m$ and $l$ have, however, quite similar slopes, and scattering at the resonances can also affect the orientation of the ridge with respect to the ARL in action space. Sellwood (2010) therefore proposed to use angle space to constrain the $m$ and $l$ of this resonance. In particular, he suggested to use the relation

$m \theta_{\phi}+l \theta_{R} \simeq$ const. $\Longrightarrow \frac{\Delta \theta_{\phi}}{\Delta \theta_{R}}=-\frac{m}{l}$

for stars trapped at a resonance (see Section 2.2). From analysing data from the Geneva-Copenhagen Survey within 200 pc (GCS; Holmberg et al. 2009), he concluded that stars in the Hyades ridge lie along $\Delta \theta_{\phi} / \Delta \theta_{R}>0$, being indicative of an inner Lindblad resonance of a slowly rotating spiral arm.

However, McMillan (2011) demonstrated that this conclusion was mainly driven by selection effects: Firstly, the asymmetric drift in the Solar neighbourhood lead to a general excess of stars close to apocenter. Secondly, the limited survey volume restricted the observable region in angle plane predominantly to the two diagonally opposite quadrants ('outward-moving' $\wedge \theta_{\phi}<0$ ) and ('inward-moving' $\wedge \theta_{\phi}>0$ ). ${ }^{11}$ Together, these biases lead to measurements of $\Delta \theta_{\phi} / \Delta \theta_{R}>0$ for the 'Hyades' stars.

Hahn et al. (2011) revisited the analysis using a different set of stars within 200pc, taken from the RAVE and SDSS surveys (Zwitter et al. 2008; Abazajian et al. 2009), confirming the claim by Sellwood (2010) that the 'Hyades' look like a resonant ridge in action space, and the conclusion by McMillan (2011) that the severe selection effects do not allow to distinguish between outer and inner Lindblad resonance and to determine $m$.

These authors have selected their candidates for stars scattered at a Lindblad resonance along the ARL in action space. This makes sense for an inner Lindblad resonance, given the alignment of the scattering direction with the ARL (c.f. fig. 5 in Sellwood 2010). For the OLR, we expect the resonant $x_{1}(1)$ orbit stars to be scattered on average off the ARL, towards higher $L_{z}$, while the $x_{1}(2)$ orbits lie on the lower- $L_{z}$ side of the ARL. In this work, where we have looked only at the OLR with $m=2$, we have explicitly made this distinction between the two orbit classes and used it as an additional diagnostic to identify the OLR in Appendix C1.

These authors have also stated that it is because of the smallness of the $200 \mathrm{pc}$ Solar neighbourhood that it is difficult to determine the exact $m$ of the resonance based on a search for $\Delta \theta_{R} / \Delta \theta_{\phi}=-m / l$ features. In this work, thanks to the $3 \mathrm{kpc}$ extent of Gaia DR2, we were able to search specifically for the OLR with $m=2$. Based on our test particle simulations, we found that this OLR is actually the resonance for which this strategy can work best.

Concerning the 'Hyades' - the main interest of Sellwood (2010), McMillan (2011, 2013), and Hahn et al. (2011)—we first identified that it could be related to the bar's OLR based on the slope in angle space for $\Omega_{\mathrm{bar}}=1.74 \Omega_{0}$ (in Section 4.2 and Figure B1), but given the evolution of this signature that we expect with $L_{z}$ (or assumed $\Omega_{\text {bar }}$; in Appendices C1- C2, Figure C1), we rule this candidate out. In Section 5.3 and Figure 9, we speculated that the 'Hyades' could be related to the bar's 1:4 resonance. The work by Monari et al. (2019a) suggests the 'Hyades/Horn' to be close to the bar's 1:6 resonance. In the past, some other authors (Quillen \& Minchev 2005; Pompéia et al. 2011) have suggested that the 'Hyades' could be caused by the $-1: 4$ inner Lindblad resonance of a spiral arm. Angle space in the corresponding panels in Figure B1 does not obviously exhibit a feature of slope of $-m / l=+4$ or $-m / l=-6$ clearly distinct from the ' $\langle\phi\rangle \sim 0$ ' line (which itself exhibits slopes of -4 and +6 ), so we can neither support nor contradict this. The resonant origin of the 'Hyades' remains therefore an open question.

\subsection{Discussion of previous work on angle asymmetries in Gaia DR2}

Our study is based on comparing stellar numbers at positive and negative $\left(\theta_{\phi}, \theta_{R}\right)$ within $\sim L_{z}$ bins to uncover the characteristic orbit shape at the OLR. A recent study by Hinkel et al. (2020b) compared in the Gaia DR2 data stellar numbers at positive and negative Galactic azimuth $\phi$ within $R$ bins. They searched for a number asymmetry flip at $R_{\mathrm{OLR}}$ induced along $\phi$ by the OLR's orbit shape and orientation flip (c.f., e.g., Figure A1 in Paper I). Because

$11 \theta_{R}$ is periodic in $2 \pi$, and usually presented as $\theta_{R} \in[-\pi, \pi]$. Sellwood (2010), McMillan (2011), and Hahn et al. (2011) define $\theta_{R}=0$ at apocenter and $\theta_{R}<0$ is therefore 'outward-moving'. We use the definition from galpy, where $\theta_{R}=0$ at pericenter, and $\theta_{R}>0$ denotes therefore 'outwardmoving'. This zero-point shift leaves Equation (33) unchanged. 
of the relations between $\theta_{\phi} \longleftrightarrow \phi$ and $L_{z} \longleftrightarrow R$, respectively, the basic idea in these two works is very similar.

Hinkel et al. (2020b) found $\Omega_{\mathrm{bar}}=49.3 \pm 2.2 \mathrm{~km} / \mathrm{s} / \mathrm{kpc}=$ $1.75 \pm 0.08 \Omega_{0}$ (for $\Omega_{0}=(229.0 \mathrm{~km} / \mathrm{s}) /(8.122 \mathrm{kpc})$; Eilers et al. 2019 ) as their best candidate. This is consistent with the classic fast Hercules bar and our weak candidate from angle space with $\Omega_{\text {bar }}=1.74-1.77 \Omega_{0}$ (Figures 8 and D1).

Our study included the radial phase $\theta_{R}$ as an additional piece of information in the analysis. This changed the outcome of the asymmetry study significantly: It ruled out the fast Hercules bar, and introduced the OLR of a bar with pattern speed $1.4 \Omega_{0}$ as a strong candidate, even though it misses the classic OLR orbit orientation flip.

\section{SUMMARY AND CONCLUSION}

In this work, we propose an independent strategy to identify the Outer Lindblad Resonance (OLR; $l=+1, m=2$ ) of the Galactic bar in the Gaia DR2 RVS data within $3 \mathrm{kpc}$, using the space of orbital angles $\left(\theta_{\phi}, \theta_{R}\right)$ (Figure 1) and the relation

$\theta_{R}=-2\left(\theta_{\phi}-\phi_{\text {bar }}\right)+\pi$,

(with slope $\Delta \theta_{R} / \Delta \theta_{\phi}=-m / l=-2$ ) characteristic for resonant orbits of the $x_{1}(1)$ OLR orbit class aligned with the bar (Figures 2-3).

Based on idealized test particle simulations within a barred galaxy potential, we illustrate that this diagnostic property can be made visible for the following sub-selection of stars: For the true pattern speed $\Omega_{\mathrm{bar}}, x_{1}(1)$ orbits dominate the OLR's resonant scattering ridge in action space $\left(L_{z}, J_{R}\right)$ (on the high- $L_{z}$ side of the resonance line; Figures 4-5).

The completeness of surveys decreases with distance from the Sun. This effectively hides the resonant angle signatures. We show that by investigating the relative number difference $\delta N\left(\theta_{\phi}, \theta_{R}\right)$ of stars at $\left(\theta_{\phi}, \theta_{R}\right)$ vs. $\left(-\theta_{\phi},-\theta_{R}\right)$, we can mitigate this selection effect and reveal the OLR's angle slope, as $\delta N=0$ along the line $\theta_{R}=-2 \theta_{\phi}$ (Figure 6).

This strategy can be affected if the survey's completeness also exhibits differences at Galactic longitudes $\pm l$ (i.e. at $+|l|$ vs. $-|l|$ ). The OLR signature should still be visible for completeness differences of less than $30 \%$, as is the case in the Gaia DR2 RVS data. We find that marking the line along which the mean Galactic azimuth $\langle\phi\rangle \sim 0$ in the $\left(\theta_{\phi}, \theta_{R}\right)$ plane is a good diagnostic to gauge if the patterns in $\delta N$ in the angle plane could be affected by selection effects (Figure 7).

We present the Gaia DR2 RVS angle data out to $3 \mathrm{kpc}$ as a function of $L_{z}$-or similarly, as a function of the location of the OLR in action space (Figure B1). This reveals phase-substructure that might be informative about the physical mechanisms causing the features at this location in action space.

By comparing this to the expected OLR $x_{1}(1)$ signature $(\delta N=$ 0 along $\theta_{R}=-2 \theta_{\phi}$ ), we extract several candidates for the MW's true OLR (Figure 8):

$\Omega_{\text {bar }} \sim 1.2-1.27 \Omega_{0} \sim 33-36 \mathrm{~km} / \mathrm{s} / \mathrm{kpc}$ explains the 'Hat' moving group with the OLR of a slow bar. It is consistent with a flip from outward-to-inward movements, classically expected at the OLR. However, the outward-moving part does not exhibit the angle signatures expected for the associated $x_{1}(2)$ orbits, anti-aligned with the bar (Figure $\mathrm{C} 1$ ). We note that the selection effects around this OLR radius $\left(R_{\mathrm{OLR}} \sim 10.5-10.7 \mathrm{kpc}\right)$ are quite strong, affecting our conclusions. A larger coverage of the Galactic disk with precise phase-space data should resolve this. This pattern speed is a favourite in the literature as it associates the 'Hercules' stream with the bar's CR resonance.

$\Omega_{\mathrm{bar}} \sim 1.4-1.45 \Omega_{0} \sim 39-41 \mathrm{~km} / \mathrm{s} / \mathrm{kpc}$ is the most convincing candidate in angle space. It would associate part of the 'Sirius' moving group with the OLR of a slightly faster slow bar and agrees interestingly with measurements from the Galactic center. For this pattern speed, the outward-moving part of the OLR signature with $x_{1}(2)$ orbits is missing, however. If this was the true OLR, we require a dynamical mechanism that could explain the absence of $x_{1}(2)$ OLR orbits.

The angle planes at $\Omega_{\mathrm{bar}} \sim 1.6-1.65 \Omega_{0} \sim 44-47 \mathrm{~km} / \mathrm{s} / \mathrm{kpc}$ and $\Omega_{\text {bar }} \sim 1.74-1.77 \Omega_{0} \sim 48-50 \mathrm{~km} / \mathrm{s} / \mathrm{kpc}$ also show signatures reminiscent of the OLR $x_{1}(1)$ orbits, with the resonance line falling between 'Hyades' and 'Sirius', and close to the 'Hyades' and 'Horn', respectively. But when investigating the orbital phase-structure in an extended $L_{z}$ region (or equivalently $\Omega_{\text {bar }}$-OLR range in action space) around it, angle space does not agree with our expectation (c.f. Figure C1).

In addition, we show that at the OLR of the classic fast bar pattern speed, $\Omega_{\text {bar }} \sim 1.85 \Omega_{0} \sim 51 \mathrm{~km} / \mathrm{s} / \mathrm{kpc}$, which associates the 'Horn' with the $x_{1}(1)$ and 'Hercules' with $x_{1}(2)$ OLR orbits, angle space does not exhibit the OLR signature at all. This result is also robust against the influence of selection effects. We propose therefore that the Gaia DR2 angle data rules out that the MW has a fast bar (Figure 8).

We uncovered a curious coincidence in angle space related to the higher-order resonances of the strong bar candidate with $\Omega_{\mathrm{bar}}=$ $1.4 \Omega_{0}$, the slightly faster slow bar. Close to its $l: m$ resonances with $l=+1$ and $m \in\{3,4,5\}$, the angle space of Gaia DR2 exhibits features with $\delta N=0$ along lines $\theta_{R}=-m / l \cdot \theta_{\phi}$ (Figure 9). On the one hand, this could further support this pattern speed candidate, as we expect this slope in theory from the intrinsic orbit shapes. On the other hand, we could not explain this observation based on our test particle simulations. The true nature of these features remains an open question.

Gaia DR3 will provide accurate 6D phase-space measurements for stars even beyond $3 \mathrm{kpc}$. For this larger coverage of the Galactic disk, our proposed strategy to search for resonance signatures of the central bar in the space of orbital phase angles promises new insights into the dynamical mechanisms that have shaped our Galaxy.

\section{DATA AVAILABILITY}

This work has made use of data from Gaia DR2 (Gaia Collaboration et al. 2016, 2018a) available at https://gea.esac.esa.int/ archive. For Gaia DR2's radial velocity sample (Katz et al. 2019), stellar distances were taken from Schönrich et al. (2019) and are available at https : //zenodo.org/record/2557803.

Action-angle estimation and test particle simulations underlying this article were produced with the galpy code by Bovy (2015) which is publicly available at http://github.com/jobovy/ galpy. The action-angle data and simulations will be shared on reasonable request to the corresponding author.

\section{ACKNOWLEDGEMENTS}

The author thanks Jan Rybizki for providing the Gaia DR2 RVS completeness function prior to publication, Francesca Fragkoudi for sharing code to derive OLR parent orbits, Rebekka Bieri and 
Simon White for valuable comments on a first paper draft, and the MPA's Dynamics group for helpful discussions. The author thanks also the referee James Binney for instructive suggestions to improve especially the theory part of this paper.

This work has made use of data from the European Space Agency (ESA) mission Gaia (https://www. cosmos.esa.int/ Gaia), processed by the Gaia Data Processing and Analysis Consortium (DPAC, https://www.cosmos.esa.int/web/ Gaia/dpac/consortium). Funding for the DPAC has been provided by national institutions, in particular the institutions participating in the Gaia Multilateral Agreement.

This project was developed in part at the 2019 Santa Barbara Gaia Sprint, hosted by the Kavli Institute for Theoretical Physics at the University of California, Santa Barbara.

This research was supported in part at KITP by the HeisingSimons Foundation and the National Science Foundation under Grant No. NSF PHY-1748958.

\section{REFERENCES}

Abazajian K. N., et al., 2009, ApJS, 182, 543

Antoja T., Valenzuela O., Pichardo B., Moreno E., Figueras F., Fernández D., 2009, ApJ, 700, L78

Antoja T., et al., 2014, A\&A, 563, A60

Asano T., Fujii M. S., Baba J., Bédorf J., Sellentin E., Portegies Zwart S., 2020, MNRAS, 499, 2416

Athanassoula E., 1992, MNRAS, 259, 328

Athanassoula E., Misiriotis A., 2002, MNRAS, 330, 35

Athanassoula E., Bienayme O., Martinet L., Pfenniger D., 1983, A\&A, 127, 349

Binney J., 2012, MNRAS, 426, 1324

Binney J., 2018, MNRAS, 474, 2706

Binney J., 2020a, MNRAS, 495, 886

Binney J., 2020b, MNRAS, 495, 895

Binney J., McMillan P., 2011, MNRAS, 413, 1889

Binney J., Spergel D., 1982, ApJ, 252, 308

Binney J., Tremaine S., 2008, Galactic Dynamics: Second Edition. Princeton University Press

Boubert D., Everall A., 2020, MNRAS, 497, 4246

Bovy J., 2015, ApJS, 216, 29

Bovy J., Rix H.-W., 2013, ApJ, 779, 115

Bovy J., Leung H. W., Hunt J. A. S., Mackereth J. T., García-Hernández D. A., Roman-Lopes A., 2019, MNRAS, 490, 4740

Buta R., Laurikainen E., Salo H., Block D. L., Knapen J. H., 2006, AJ, 132, 1859

Chakrabarty D., 2007, A\&A, 467, 145

Chiba R., Schönrich R., 2021, MNRAS, 505, 2412

Chiba R., Friske J. K. S., Schönrich R., 2021, MNRAS, 500, 4710

Clarke J. P., Wegg C., Gerhard O., Smith L. C., Lucas P. W., Wylie S. M., 2019, MNRAS, 489, 3519

Combes F., Sanders R. H., 1981, A\&A, 96, 164

Contopoulos G., Grosbol P., 1989, A\&ARv, 1, 261

D’Onghia E., L. Aguerri J. A., 2020, ApJ, 890, 117

De Simone R., Wu X., Tremaine S., 2004, MNRAS, 350, 627

Dehnen W., 2000, AJ, 119, 800

Eilers A.-C., Hogg D. W., Rix H.-W., Ness M. K., 2019, ApJ, 871, 120

Famaey B., Pont F., Luri X., Udry S., Mayor M., Jorissen A., 2007, A\&A, 461, 957

Fragkoudi F., Athanassoula E., Bosma A., Iannuzzi F., 2015, MNRAS, 450, 229

Fragkoudi F., et al., 2019, MNRAS, 488, 3324

Fragkoudi F., et al., 2020, MNRAS, 494, 5936

Friske J. K. S., Schönrich R., 2019, MNRAS, 490, 5414

Fujii M. S., Bédorf J., Baba J., Portegies Zwart S., 2019, MNRAS, 482, 1983
Fux R., 2001a, A\&A, 373, 511

Fux R., 2001b, A\&A, 373, 511

Gaia Collaboration et al., 2016, A\&A, 595, A1

Gaia Collaboration et al., 2018a, A\&A, 616, A1

Gaia Collaboration et al., 2018b, A\&A, 616, A11

Gaia Collaboration et al., 2021, A\&A, 649, A1

Gravity Collaboration et al., 2018, A\&A, 615, L15

Hahn C. H., Sellwood J. A., Pryor C., 2011, MNRAS, 418, 2459

Harsoula M., Kalapotharakos C., 2009, MNRAS, 394, 1605

Hinkel A., Gardner S., Yanny B., 2020a, ApJ, 893, 105

Hinkel A., Gardner S., Yanny B., 2020b, ApJ, 899, L14

Holmberg J., Nordström B., Andersen J., 2009, A\&A, 501, 941

Hunt J. A. S., Bovy J., 2018, MNRAS, 477, 3945

Hunt J. A. S., Kawata D., Monari G., Grand R. J. J., Famaey B., Siebert A., 2017, MNRAS, 467, L21

Hunt J. A. S., Bub M. W., Bovy J., Mackereth J. T., Trick W. H., Kawata D., 2019, MNRAS, 490, 1026

Hunt J. A. S., Johnston K. V., Pettitt A. R., Cunningham E. C., Kawata D., Hogg D. W., 2020, MNRAS, 497, 818

Jurić M., et al., 2008, ApJ, 673, 864

Kaasalainen M., 1994, MNRAS, 268, 1041

Kalnajs A. J., 1991, in Sundelius B., ed., Dynamics of Disc Galaxies. p. 323

Katz D., et al., 2019, A\&A, 622, A205

Kawata D., Baba J., Ciucă I., Cropper M., Grand R. J. J., Hunt J. A. S., Seabroke G., 2018, MNRAS, 479, L108

Kawata D., Baba J., Hunt J. A. S., Schönrich R., Ciucă I., Friske J., Seabroke G., Cropper M., 2021, MNRAS, 508, 728

Khanna S., et al., 2019, MNRAS, 489, 4962

Khoperskov S., Gerhard O., Di Matteo P., Haywood M., Katz D., Khrapov S., Khoperskov A., Arnaboldi M., 2020, A\&A, 634, L8

Laporte C. F. P., Minchev I., Johnston K. V., Gómez F. A., 2019, MNRAS, 485,3134

Laskar J., 1993, Celestial Mechanics and Dynamical Astronomy, 56, 191

Lynden-Bell D., 1979, MNRAS, 187, 101

McMillan P. J., 2011, MNRAS, 418, 1565

McMillan P. J., 2013, MNRAS, 430, 3276

Michtchenko T. A., Lépine J. R. D., Pérez-Villegas A., Vieira R. S. S., Barros D. A., 2018, ApJ, 863, L37

Minchev I., Famaey B., 2010, ApJ, 722, 112

Minchev I., Boily C., Siebert A., Bienayme O., 2010, MNRAS, 407, 2122

Monari G., Famaey B., Siebert A., Grand R. J. J., Kawata D., Boily C., 2016, MNRAS, 461, 3835

Monari G., Famaey B., Siebert A., Duchateau A., Lorscheider T., Bienaymé O., 2017a, MNRAS, 465, 1443

Monari G., Kawata D., Hunt J. A. S., Famaey B., 2017b, MNRAS, 466, L113

Monari G., Famaey B., Fouvry J.-B., Binney J., 2017c, MNRAS, 471, 4314

Monari G., Famaey B., Siebert A., Wegg C., Gerhard O., 2019a, A\&A, 626, A41

Monari G., Famaey B., Siebert A., Wegg C., Gerhard O., 2019b, A\&A, 626, A41

Monari G., Famaey B., Siebert A., Bienaymé O., Ibata R., Wegg C., Gerhard O., 2019c, A\&A, 632, A107

Mühlbauer G., Dehnen W., 2003, A\&A, 401, 975

Ohta K., 1996, in Buta R., Crocker D. A., Elmegreen B. G., eds, Astronomical Society of the Pacific Conference Series Vol. 91, IAU Colloq. 157: Barred Galaxies. p. 37

Pérez-Villegas A., Portail M., Wegg C., Gerhard O., 2017, ApJ, 840, L2

Pettitt A. R., Ragan S. E., Smith M. C., 2020, MNRAS, 491, 2162

Pfenniger D., Friedli D., 1991, A\&A, 252, 75

Pompéia L., et al., 2011, MNRAS, 415, 1138

Portail M., Gerhard O., Wegg C., Ness M., 2017, MNRAS, 465, 1621

Quillen A. C., 2003, AJ, 125, 785

Quillen A. C., Minchev I., 2005, AJ, 130, 576

Quillen A. C., Frogel J. A., Gonzalez R. A., 1994, ApJ, 437, 162

Quillen A. C., Dougherty J., Bagley M. B., Minchev I., Comparetta J., 2011, MNRAS, 417, 762

Quillen A. C., et al., 2018, MNRAS, 480, 3132 
Reid M. J., et al., 2014, ApJ, 783, 130

Rybizki J., Drimmel R., 2018, gdr2_completeness: GaiaDR2 data retrieval and manipulation (ascl:1811.018)

Rybizki J., Rix H.-W., Demleitner M., Bailer-Jones C. A. L., Cooper W. J., 2021, MNRAS, 500, 397

Sanders J. L., Smith L., Evans N. W., 2019, MNRAS, 488, 4552

Schönrich R., Binney J., Dehnen W., 2010, MNRAS, 403, 1829

Schönrich R., McMillan P., Eyer L., 2019, MNRAS, 487, 3568

Sellwood J. A., 2010, MNRAS, 409, 145

Sellwood J. A., 2012, ApJ, 751, 44

Sellwood J. A., Binney J. J., 2002, MNRAS, 336, 785

Sellwood J. A., Wilkinson A., 1993, Reports on Progress in Physics, 56, 173

Sellwood J. A., Trick W. H., Carlberg R. G., Coronado J., Rix H.-W., 2019, MNRAS, 484, 3154

Skrutskie M. F., et al., 2006, AJ, 131, 1163

Sormani M. C., Binney J., Magorrian J., 2015, MNRAS, 454, 1818

Tian H.-J., et al., 2017, Research in Astronomy and Astrophysics, 17, 114

Tremaine S., Weinberg M. D., 1984, ApJ, 282, L5

Trick W. H., Coronado J., Rix H.-W., 2019, MNRAS, 484, 3291

Trick W. H., Fragkoudi F., Hunt J. A. S., Mackereth J. T., White S. D. M., 2021, MNRAS, 500, 2645

Weinberg M. D., 1994, ApJ, 420, 597

Wheeler A., Abril-Cabezas I., Trick W. H., Fragkoudi F., Ness M., 2021, arXiv e-prints, p. arXiv:2105.05263

Xu Y., et al., 2016, Science Advances, 2, e1600878

Zwitter T., et al., 2008, AJ, 136, 421

\section{APPENDIX A: ILLUSTRATING PARENT, LIBRATING, AND CIRCULATING ORBITS AROUND THE OLR}

In Section 2, we summarized the behaviour of resonant orbits in a barred Galaxy potential in action-angle space. Figure A1 illustrates this now further with a few example orbits close to the OLR. We use the bar potential from the Fiducial_40 test particle simulation (Section 3.1) with pattern speed $\Omega_{\mathrm{bar}}=40 \mathrm{~km} / \mathrm{s} / \mathrm{kpc}$.

\section{A1 Resonant orbits}

Based on the algorithm by Fragkoudi et al. (2015) (see also Athanassoula 1992; Sellwood \& Wilkinson 1993), we selected a parent orbit at the OLR with $l=+1$ and $m=2$ from the $x_{1}(1)$ orbit class, which is shown in purple in Figure A1. Panels (h) and (j) show the fast Fourier transform (FFT) spectra of the parent orbit. Each has one dominant frequency, $\Omega_{R \text {,true }}$ and $\Omega_{\phi \text {,true }}$, in $\Omega_{R \text {,FFT }}$ and $\Omega_{\phi \text {,FFT }}$, respectively, which satisfy the resonance condition in Equation (1). For the parent orbit, $\Omega_{i \text {,true }} \approx \Omega_{i \text {,axi }}$, i.e. it lives almost exactly on top of the ARL (Equation 6) in action space (Panel (c)). This example parent orbit is launched from a position with initial radius $R_{\text {parent }}=10.89 \mathrm{kpc}$ along the $x_{\mathrm{CRF}}$ axis, its Jacobi energy is $E_{\text {Jacobi }}=-112,553 \mathrm{~km}^{2} / \mathrm{s}^{2}$ and $J_{z} \approx 0$. Different choices of $E_{\text {Jacobi }}$ would position the closed periodic orbits higher or lower on the ARL and at larger or smaller initial $R$ (see Paper I, fig. 3). Larger $J_{z}$ would move the parent orbit together with the ARL to lower $L_{z}$ (see Paper I, §5.2.2).

All orbits in this figure have the same $E_{\mathrm{Jacobi}}$, but slightly shifted initial position $R=f \times R_{\text {parent }}$ (black crosses in Panels (a) and (b)).

We show librating resonant OLR orbits in lighter shades of pink and purple in Figure A1. We used $f \in 1+\{0.04,0.09,0.12\}$ to initialize them; orbits with $f \in 1-\{0.04,0.09,0.12\}$ would look almost identical. They librate around their parent orbit with successively larger amplitude in actions, angles, and pericenter. The grey dotted line in Panel (c) denotes a line of constant fast action. The primary frequencies of the librating orbits are the same as those of the parent orbit, i.e. they satisfy Equation (1) exactly. As opposed to the parent orbit, their FFT spectra exhibit also secondary frequencies which are related to the libration (Panels (h) and (j); see also Binney \& Spergel 1982).

\section{A2 Circulating orbits}

The orbits in shades of green in Figure A1 were initialized with larger $f \in\{1.185,0.82\}$. Their pericenters are not trapped anymore in $\phi_{\mathrm{CRF}}$, but rather circulating around the bar. This can also be seen in Panels (e) and (f) for the angle coordinate $\theta_{\phi}$ with respect to the bar. The dark green orbit lives outside of the OLR and its symmetry is aligned with the bar; the light green orbit lives inside of the OLR and its symmetry is anti-aligned with the bar. We are seeing here the orbit orientation flip at the OLR. Also in action space, Panel (c), the circulating orbits are not trapped around the ARL anymore and truly live at $L_{z} \mathrm{~S}$ inside or outside of the resonance. They have clear primary frequencies $\Omega_{i, \text { true }}$ and no substantial secondary frequencies. The primary frequency 'jumps' from the librating orbits with the exact resonance frequencies to the circulating orbits whose $\Omega_{i, \text { true }}$ are close to the former's secondary libration frequencies (Binney \& Spergel 1982). The trapping of the resonant orbits therefore manifests also in frequency space.

Special cases. For close-to-circular orbits $J_{R} \sim 0$ (Binney 2020a) and in the small transition region from librating and to circulating orbits, the behaviour is more complex (e.g. Binney \& Spergel 1982), but beyond the scope of this paper.

\section{APPENDIX B: THE FULL ANGLE SPACE OF GAIA DR2 RVS: SEARCHING FOR THE OLR}

In Figure B1, we present the full 4D action-angle data of the Gaia DR2 RVS sample.

In the upper left panel, we mark the action overdensity ridges, i.e., the orbit substructures associated with the well-known moving groups in the Solar neighbourhood (T19). Each 2D bin in action space has a different 2D angle distribution. We reduce this complexity to $3 \mathrm{D}$, by binning the $\left(L_{z}, J_{R}\right)$ plane into slices roughly parallel to the action ridges. The slices are parameterized in terms of the OLR ARL position of an assumed $\Omega_{\mathrm{bar}}$ (see Section 4.2 for details).

Each angle space panel in Figure B1 contains the stars from one of these actions slices. We show the stellar number asymmetry $\delta N\left(\theta_{\phi}, \theta_{R}\right)$ (Equation 29) to make substructure better visible (see Section 3.3). Overplotted in grey is the ' $\langle\phi\rangle \sim 0$ ' line that indicates that some features might still be related to selection effects (see Section 3.4).

To help with the orientation in Gaia's angle space-which angle panel belongs to which region in action space-we provide in Figure B1:

(i) for each row of angle panels on the very right the rough $L_{z^{-}}$ range (at $J_{R} \sim 0$ ) that stars in these slices of action-angle space have.

(ii) for each angle panel the assumed $\Omega_{\mathrm{bar}}$ in units of $\Omega_{0}$ for which the respective action slice would fall together with the location of the OLR ridge.

(iii) for some angle panels a coloured dot in the upper right, which marks that this slice contains stars from one of the famous orbit substructures. 

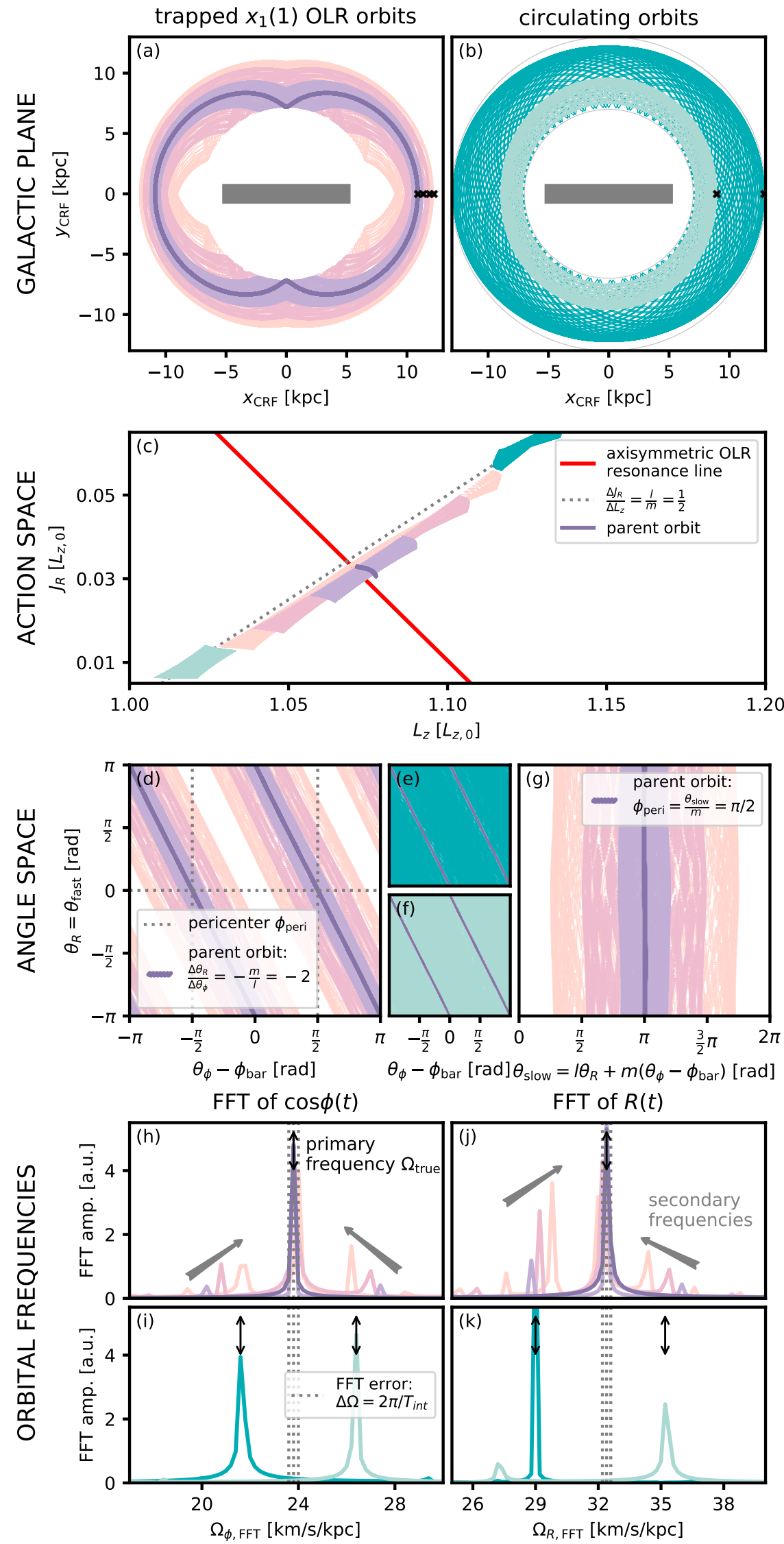

Figure A1. Comparing trapped resonant orbits at the OLR with circulating orbits in the Galactic disk plane co-rotating with the bar, as well as in action-anglefrequency space. For more details see Section 2.2 and Appendix A. 

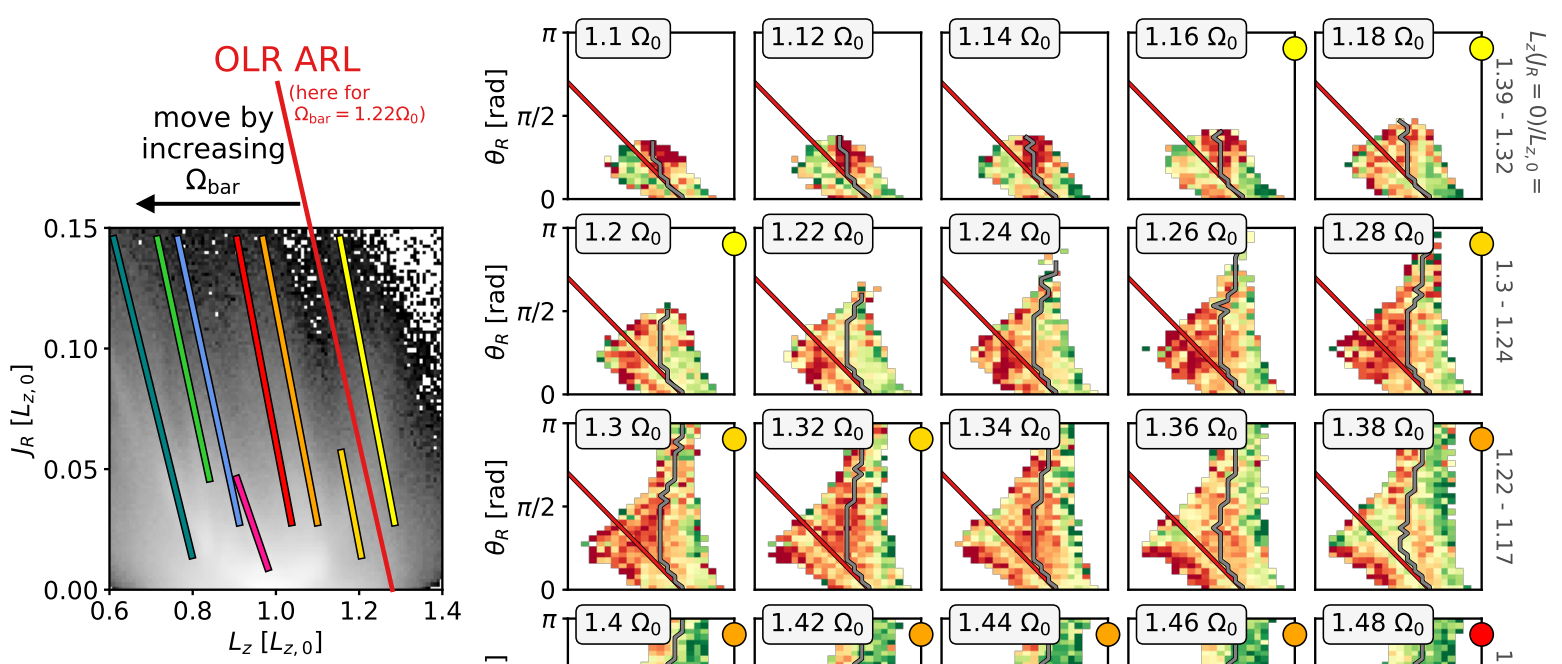

Named orbit substructure
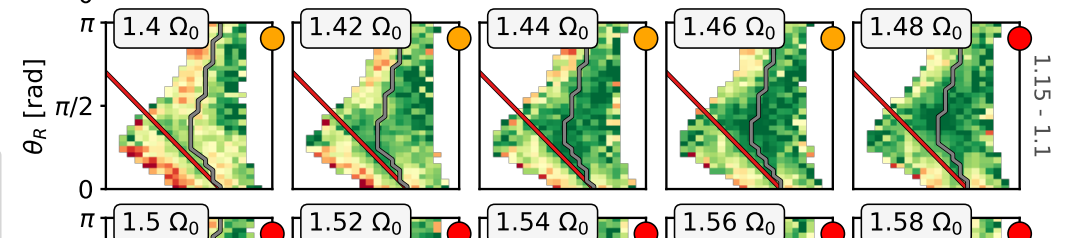

Hat (I)

O $(\mathrm{H})$

Sirius (G1)

Sirius (F1)

Hyades/Horn (E1)

Hercules/Horn (D1)

Hercules (C1)

Hercules (B)

Model prediction for the true OLR
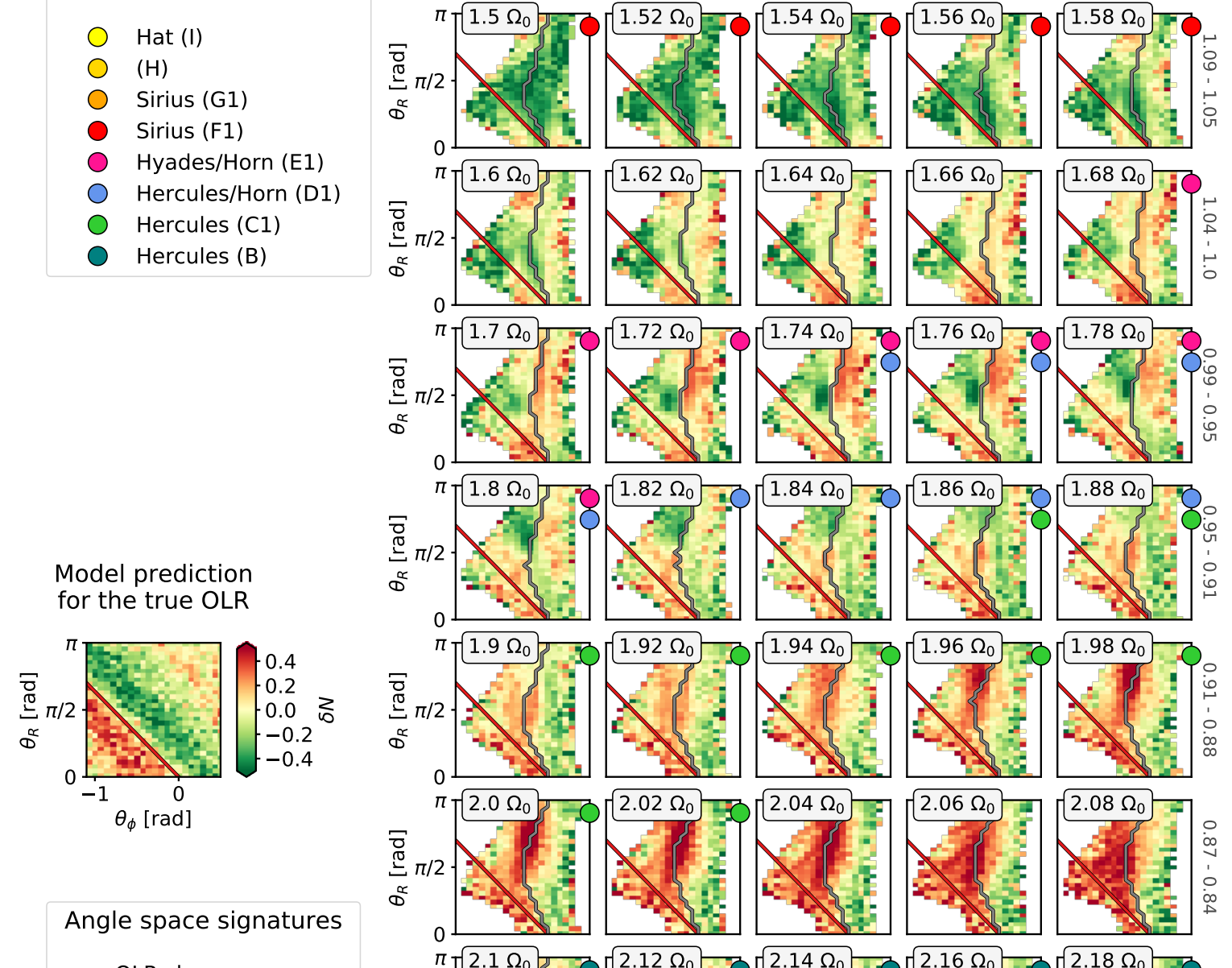

Angle space signatures

OLR slope

$-\Delta \theta_{R} / \Delta \theta_{\phi}=-m / l=-2$ Selection effect guide line, $\langle\phi\rangle \sim 0$
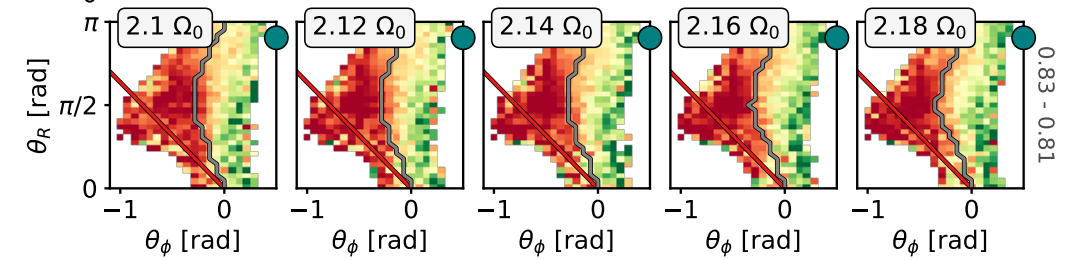

Figure B1. Searching for OLR candidates in the Gaia DR2 RVS angle data. Varying the bar pattern speed $\Omega_{\text {bar }}$ moves the OLR ARL across the action plane (upper left panel). We show the angle space asymmetry $\delta N$ for stars next to the OLR ARL for different $\Omega_{\text {bar }}($ right panels). We compare the $\delta N$ in each panel to the prediction from the simulation (lower left panel). For a detailed discussion of the most promising OLR candidates, see Figure 8. 
For example, in the panels marked by the pink dot, standing for the Hyades/Horn (E1, pink) ridge, we see an outward-moving ('red') clump at $\theta_{R} \sim 0.6 \pi$, which contains stars from the 'Hyades' moving group. The inward-moving ('green') clump at $\theta_{R} \sim \pi / 2$ in the same panels is the large scale analogue of the 'Horn' feature (around $(U, V) \sim(60,-25) \mathrm{km} / \mathrm{s})$ in local velocity space.

As laid out in Section 4.2, Figure B1 provides the basis to search for candidates for the MW's true bar OLR.

\section{APPENDIX C: FURTHER CONSTRAINTS ON BAR RESONANCES FROM ANGLE SPACE}

The OLR is the strongest signature of the bar outside of CR. In this work, we focused therefore on finding the signature of resonant $x_{1}$ (1) OLR orbits in angle space. Based on our idealized test particle simulations, the angles can be used as a diagnostic even beyond that. In the following, we will therefore shortly discuss (i) antialigned $x_{1}(2)$ orbits close to the OLR (Section C1), and (ii) the strength of the OLR signature (Section C2), (iii) 1:1 resonant orbits (Section C3), (iv) CR orbits (Section C4), and (iv) 1:4 resonant orbits (Section C5).

\section{C1 The OLR's orbit orientation flip in angle space}

Following the discussion in Sections 5.1-5.2, we expect that the OLR's classic orbit orientation flip should not only show up in action- $v_{R}$ space, but also in angle- $\delta N$ space. This provides an additional diagnostic for the bar's OLR.

In Figure C1, right-most column, we show for the Fiducial_40 and Fiducial_51 simulations angle space for stars that were taken to the left of the true OLR ridge from the outwardmoving, underdense part of the OLR signature. (We do this by moving the OLR ARL by $\Delta \Omega_{\text {bar }}=+0.15 \Omega_{0}$ with respect to the true $\Omega_{\mathrm{bar}}$.) This selection contains many stars on circulating orbits that are elongated in an anti-aligned fashion with respect to the bar with a 1:2 symmetry and belong to the $x_{1}(2)$ orbit class. The corresponding angle plane exhibits a 'green/red' pattern with OAS -2 , just as the $x_{1}(1)$ orbits in the OLR ridge (4th column in Figure $\mathrm{C} 1$ ), but with the colours reversed.

Consequently, we now search in the Gaia data in Figure B1 for a panel that is 'green' below the OAS line and 'red' above it.

The $1.1-1.14 \Omega_{0}$ panels are the only candidate. However, this is on the wrong side of the Hat bar's OLR ARL-i.e. $\Delta \Omega<0$ instead of the required $\Delta \Omega>0$ - to support the candidate $\Omega_{\mathrm{bar}}=1.2 \Omega_{0}$. This part of phase-space is also strongly affected by selection effects.

The $1.46-1.58 \Omega_{0}$ panels do have $\Delta \Omega>0$ with respect to our strong candidate $\Omega_{\mathrm{bar}}=1.4 \Omega_{0}$. But while there is 'green' below the OAS line, there is no 'red' feature above the line. This is the angle equivalent of the missing outward-moving 'red' feature in action- $v_{R}$ space for this pattern speed from Paper I, $\$ 6.3 .4$ and also shown in Figure 8.

In conclusion, we do not find any signatures of $x_{1}(2)$ OLR orbits in the Gaia angle space.

\section{C2 Strength of the OLR signature in angle space}

The strength of the bar determines how large the region in action space is within which stars are on trapped OLR $x_{1}(1)$ orbits. This region is bounded by the separatrix and determines the width of the action-space scattering ridge. The OAS signature should be visible across the whole ridge (c.f. Figure 4).
A shift in the assumed $\Omega_{\mathrm{bar}}$ of $\Delta \Omega=+0.02 \Omega_{0}$, as used for the Gaia angle exploration in Figure B1, corresponds in the OLR ARL to a shift of $\Delta L_{z} \sim-0.01 L_{z, 0} .{ }^{12}$ This is smaller than the width of the OLR ridge we observe in the simulations, $\left|\Delta L_{z}\right| \sim 0.05 L_{z, 0}$. We therefore expect to see the OLR angle signature in a range of panels in Figure B1.

In the simulations, we even observed the OLR signature over ranges of

$$
\begin{aligned}
& \text { - } \Omega_{\text {bar }}=\Omega_{\text {bar,true }}^{+0.02}(\text { Fiducial_40) and } \\
& \text { - } \Omega_{\text {bar }}=\Omega_{\text {bar,true }}^{+0.01} \text { (Fiducial_51). }
\end{aligned}
$$

The range in the Fiducial_51 simulation is larger, because we chose the bars in both simulations to have the same strength at $R_{0}=8 \mathrm{kpc}$. Consequently, the bar influence is stronger at $R_{\mathrm{OLR}}(51 \mathrm{~km} / \mathrm{s} / \mathrm{kpc})=7.3 \mathrm{kpc}$ than at $R_{\mathrm{OLR}}(40 \mathrm{~km} / \mathrm{s} / \mathrm{kpc})=9 \mathrm{kpc}$. To illustrate this, we show in the 3 rd column of Figure $\mathrm{C} 1$ the angle planes for $\Delta \Omega=-0.15 \Omega_{0} \sim-4 \mathrm{~km} / \mathrm{s} / \mathrm{kpc}$ for both simulations. The stronger the bar at $R_{\mathrm{OLR}}$, the more pronounced the scattering ridge, the larger the assumed $\Omega_{\text {bar }}$ range over which the OLR signature should show up in angle space.

This has two implications.

Firstly, this introduces an intrinsic uncertainty in our anglebased method to measure the bar's pattern speed. For the pattern speed candidates identified in Section 4.2, we adopt based on Figure B1 the following rough ranges of:

$$
\begin{aligned}
& \Omega_{\text {bar }}=(1.2 \pm 0.02) \Omega_{0} \\
& \Omega_{\text {bar }}=(1.4 \pm 0.04) \Omega_{0} \\
& \Omega_{\text {bar }}=(1.6 \pm 0.02) \Omega_{0} \\
& \Omega_{\text {bar }}=(1.74 \pm 0.01) \Omega_{0}
\end{aligned}
$$

over which the OLR signature might be visible. We note again that selection effects might be obscuring part of the signature.

Secondly, the ' $L_{z}$-range over which the OAS is visible' could be used as an additional constraint to identify the true bar OLR. If we assume that the real MW bar is not much weaker than those in the simulation, the OLR signature should in fact be visible over a range of $\Omega_{\mathrm{bar}}$. This rules out the candidate $\Omega_{\mathrm{bar}}=1.74 \Omega_{0}$ : Contrary to what we see in the simulations, the 'green' stripe across angle space changes its slope from panel to panel around $\Omega_{\mathrm{bar}}=1.74 \Omega_{0}$; only in this single panel has it the correct slope of -2 .

\section{C3 The 1:1 resonance $(l=+1, m=1)$}

For a bar perturbation with $m=2$, the second strongest resonance in the disk outside of CR after the OLR is the 1:1 outer Lindblad resonance. We showed an example 1:1 orbit in Figure 2. The 1:1 resonance creates a high- $J_{R}$ scattering ridge analogous to the OLR, which can be seen, for example, in the upper left panel of Figure 5 at $L_{z} \sim 1.4 L_{z, 0}$.

If we select stars in our test particle simulations from this ridge, the expected $-m / l=-1$ slope in angle space is indeed revealed and could therefore be used to identify this resonance.

We searched for 1:1 signatures in the Gaia angle space, but did not find any convincing candidates. This might be due to this resonance occurring at larger radius than the OLR and being therefore more difficult to detect. For realistic bar pattern speeds, the 1:1

12 The shift $\Delta \Omega=+0.02 \Omega_{0}$ in $\Omega_{\text {bar }}$ corresponds to slightly different shifts $\Delta L_{z}$ of the OLR ARL for different pattern speeds: for example, $\Delta L_{z}=$ -0.017 or $-0.008 L_{z, 0}$ at $\Omega_{\mathrm{bar}}=1.2$ or $1.85 \Omega_{0}$, respectively. 

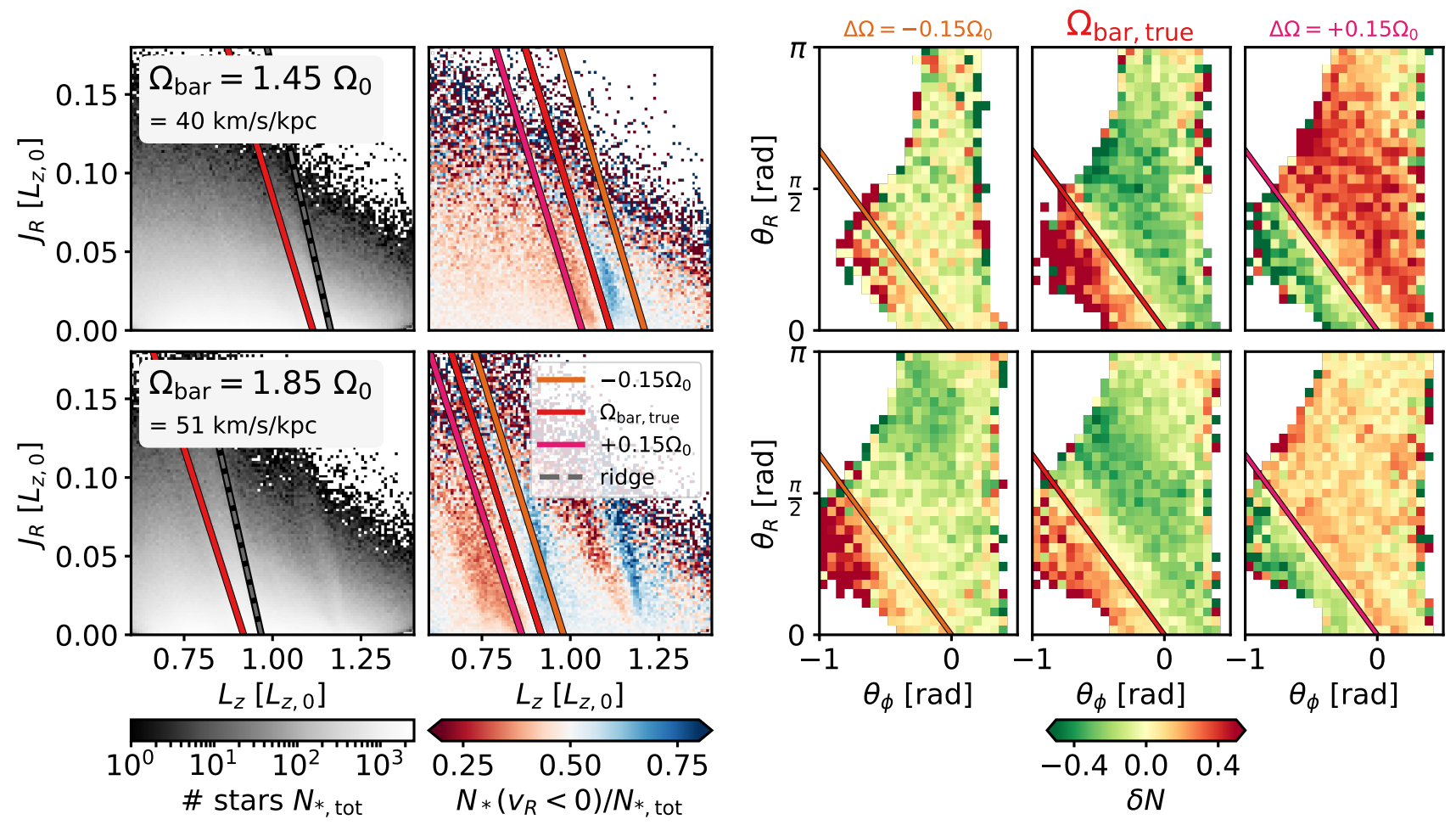

Figure C1. The orbit orientation flip at the OLR—causing the 'red/blue' feature in action space (2nd column)—can be seen in the Fiducial_40 and Fiducial_51 simulations also in angle space (4th vs. 5th column). No such angle asymmetry flip is observed in the Gaia angle data in Figure B1. Apart from this, the simulations tell us, that the OLR angle slope of -2 should be visible over a range of assumed $\Omega_{\text {bar }}(3 r d$ column). This supports the slow Hat or slightly faster slow $\mathrm{S} 19 \mathrm{~B} 19 \Omega_{\mathrm{bar}}$.

resonance falls into a region in the Galactic disk that is (a) more strongly affected by selection effects and (b) less populated by stars. If the 1:1 falls at all into the survey volume, angle space $\left(\theta_{\phi}, \theta_{R}\right)$ will be less well sampled and appear more noisy.

As mentioned in Dehnen (2000) and in Paper I, there could be —at least locally for $d<200-600 \mathrm{pc}-\mathrm{a}$ 'red/blue' feature in action space around the 1:1 resonance for the fast Hercules pattern speed. However, the corresponding angle space close to the gold $\mathrm{H}$ ) ridge in Figure B1 does not support this.

\section{C4 The co-rotation resonance $(l=0)$}

The bar's CR strongly redistributes stars in $L_{z}$, which leaves characteristic patterns in the metallicity profile of the Galactic disk (e.g. Chiba \& Schönrich 2021; Wheeler et al. 2021).

Based on our simulations, angle space, however, is not suited to identify CR. Within a survey volume around the Sun, no selection of stars based purely on action space was possible that isolated a highenough fraction of resonant CR stars to make the $-m / l=-\infty$ (i.e. vertical) angle signature visible. This is because $C R$ orbits oscillate around the bar's minor axis (Figure 2) and not many are actually visiting the Solar neighbourhood (c.f., e.g., D'Onghia \& L. Aguerri 2020; Wheeler et al. 2021).

\section{C5 The 1:4 resonance $(l=+1, m=4)$}

Orbits at the 1:4 resonance follow $\Delta \theta_{R} / \Delta \theta_{\phi}=-4$ (see Figure 2). However, the signatures that we expect in the cumulative stellar distribution around this resonance depend strongly on the choice of $m=4$ Fourier component of the bar model.

There are two aspects:

(i) Strength of the $m=4$ bar component. To create a prominent high- $J_{R}$ overdensity ridge at the 1:4 resonance in action space, our test particle simulations required $m=4$ bar components with very strong $\left|\alpha_{m=4}\right| \gtrsim 0.001$ (c.f. Monari et al. 2019a). This is consistent with the $m=4$ bar strength used in Hunt \& Bovy (2018) and Hunt et al. (2019), $\alpha_{m=4}=0.0015$, which is three times larger than the values quoted in these papers (J.A.S. Hunt, private communication).

(ii) Orientation of the $m=4$ component. Hunt \& Bovy (2018) used $\alpha_{m=4}<0$, which aligns the potential minimum of the $m=4$ bar component with the potential maximum of the $m=2$ component, creating an overall boxy bar shape. They observed a 'red/blue' feature, i.e. 'Hercules/Horn'-like outward-inward-motions, at the 1:4 resonance. ${ }^{13}$ Hunt et al. (2019, their fig. 14) explained this by two classes of 1:4 orbits: one that had pericenters close to the bar's major and minor axes (c.f. Figure $2, \theta_{\text {slow }}=0$ ), and one that was rotated by $45^{\circ}$ and had its apocenters along the bar's major and minor axes $\left(\theta_{\text {slow }}=\pi\right)$. In our analogous simulations, only the former orbit class got populated and no 1:4 'red/blue' features were observed. Fig. 4 in Fux (2001a) suggests that these are stable

13 For $\alpha_{m=4}>0$, which describes a pointy bar with ansae, Hunt \& Bovy (2018) observed a 'blue/red' feature, i.e., the opposite way around as for the classic OLR or the boxy bar 1:4 feature. 
orbits, while the $45^{\circ}$-rotated class of 1:4 orbits is unstable. It therefore depends on the kind of 1:4 orbits that get populated by stars, if and what kind of inward-/outward signature is observed at the $1: 4$ resonance.

The orientation of the $m=4$ component and knowledge about the (re-)population history of different 1:4 orbit classes are essential for an analysis in $\left(L_{z}, J_{R}, v_{R}\right)$ space. For the analysis in $\left(\theta_{\phi}, \theta_{R}, \delta N\right)$, it might not matter that much, as this method is also sensitive to the symmetry of the orbit shape, and not just to the direction in which the stars move.

In our test particle simulations with a boxy bar, we were only able to make the expected $\Delta \theta_{R} / \Delta \theta_{\phi}=-4$ signature visible in angle space, when we imposed a strong $m=4$ component with respect to the bar's basic quadrupole, e.g. $\left(\alpha_{m=2}, \alpha_{m=4}\right)=(0.02,-0.0015)$. In this case, the trapping region and libration amplitude are large enough for a substantial ridge to develop at the 1:4 ARL in action space. The transition around the angle slope line for 1:4 orbits with $\theta_{\text {slow }}=\pi$ is from 'green' to 'red', similar to the $x_{1}(2)$ orbits in Figure $\mathrm{C} 1$.

In the case of a pointy bar and 1:4 orbits with $\theta_{\text {slow }}=0$, the expected transition is from 'red' to 'green', i.e. the opposite way around.

\section{APPENDIX D: THE EFFECT OF USING A DIFFERENT GALACTIC POTENTIAL MODEL}

Estimates of action-angle coordinates depend on the assumed gravitational potential model for the MW and the Sun's location in it. In the analyses of the Gaia DR2 RVS data in this work, we have used the MWPotential2014 model by Bovy (2015), with the Sun at $R_{0}=8 \mathrm{kpc}, v_{\text {circ }}\left(R_{0}\right)=220 \mathrm{~km} / \mathrm{s}$. Figure D1 repeats this analysis for the MW potential model by Eilers et al. (2019), which uses $R_{0}=8.122 \mathrm{kpc}$ (Gravity Collaboration et al. 2018), and has $v_{\text {circ }}\left(R_{0}\right)=229.8 \mathrm{~km} / \mathrm{s}$. The pattern speed $\Omega_{\mathrm{bar}}$ is in this case given in units of $\Omega_{0}=v_{\text {circ }}\left(R_{0}\right) / R_{0}=28.3 \mathrm{~km} / \mathrm{s} / \mathrm{kpc}$. The general conclusions of this work remain unchanged when using this potential, but there are some subtle differences.

The OLR candidates identified in Section 4.2 are found at slightly different pattern speeds:

- $\Omega_{\mathrm{bar}}=1.27 \Omega_{0}=35.9 \mathrm{~km} / \mathrm{s} / \mathrm{kpc}$, with the OLR at the Hat.

- $\Omega_{\mathrm{bar}}=1.45 \Omega_{0}=41.0 \mathrm{~km} / \mathrm{s} / \mathrm{kpc}$, with the OLR on the high- $L_{z}$ edge of the Sirius ridge.

- $\Omega_{\text {bar }}=1.65 \Omega_{0}=46.7 \mathrm{~km} / \mathrm{s} / \mathrm{kpc}$, with the OLR between the 'Hyades' and 'Sirius'.

- $\Omega_{\mathrm{bar}}=1.77 \Omega_{0}=48.1 \mathrm{~km} / \mathrm{s} / \mathrm{kpc}$.

For the Eilers et al. (2019) potential model, the angle plane at $\Omega_{\mathrm{bar}} \sim 1.45 \Omega_{0}$ (slightly faster slow bar) looks even more convincing than in the MWPotential2014 (Figure 8) when comparing to the model expectation for the OLR signature with angle slope -2 . The angle plane for $\Omega_{\mathrm{bar}} \sim 1.27 \Omega_{0}$ (slow bar), on the other hand, appears noisier; but because of the selection effects, we cannot rule this candidate out yet.

The pattern speed $1.77 \Omega_{0}$ is also in the Eilers et al. (2019) potential ruled out, because of the signature's variation with $L_{z}$ as argued in Section C2.

The pattern speed of the fast bar, $\Omega_{\mathrm{bar}} \sim 1.85 \Omega_{0}$, does also in the Eilers et al. (2019) potential not exhibit the correct OLR slope and is therefore dismissed as a candidate, as discussed in Section 4.2 .
In Section 5.3 and in Figure 9, we found features in angle space with slopes of $-m / l$ at higher-order resonances $l=+1$ and $m \in\{3,4,5\}$. In this potential model, we reproduced this finding for $\Omega_{\mathrm{bar}}=1.41 \Omega_{0}=39.9 \mathrm{~km} / \mathrm{s} / \mathrm{kpc}$. The corresponding OLR in Figure D1 is however not as convincing, as it misses the 'green' part of the angle signature. The curious coincidence, that this pattern speed agreed also with the pattern speed of one of the OLR candidates, occurred therefore only for the MWPotential2014, but not for the Eilers et al. (2019) potential.

This paper has been typeset from a $\mathrm{T}_{\mathrm{E}} \mathrm{X} / \mathrm{LT}_{\mathrm{E}} \mathrm{X}$ file prepared by the author. 

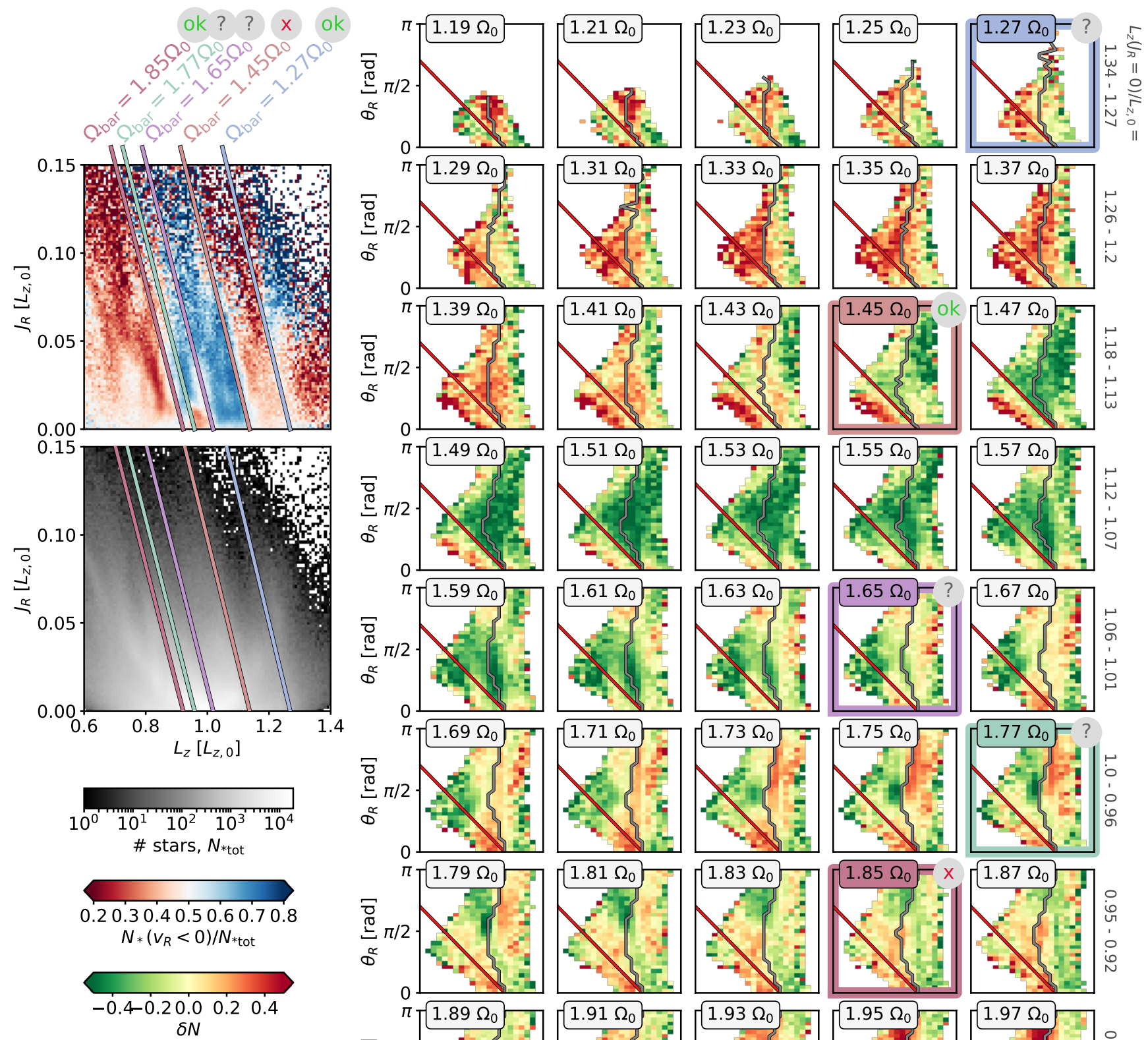

Angle space signatures

OLR slope

$\Delta \theta_{R} / \Delta \theta_{\phi}=-m / l=-2$

Selection effect

guide line, $\langle\phi\rangle \sim 0$
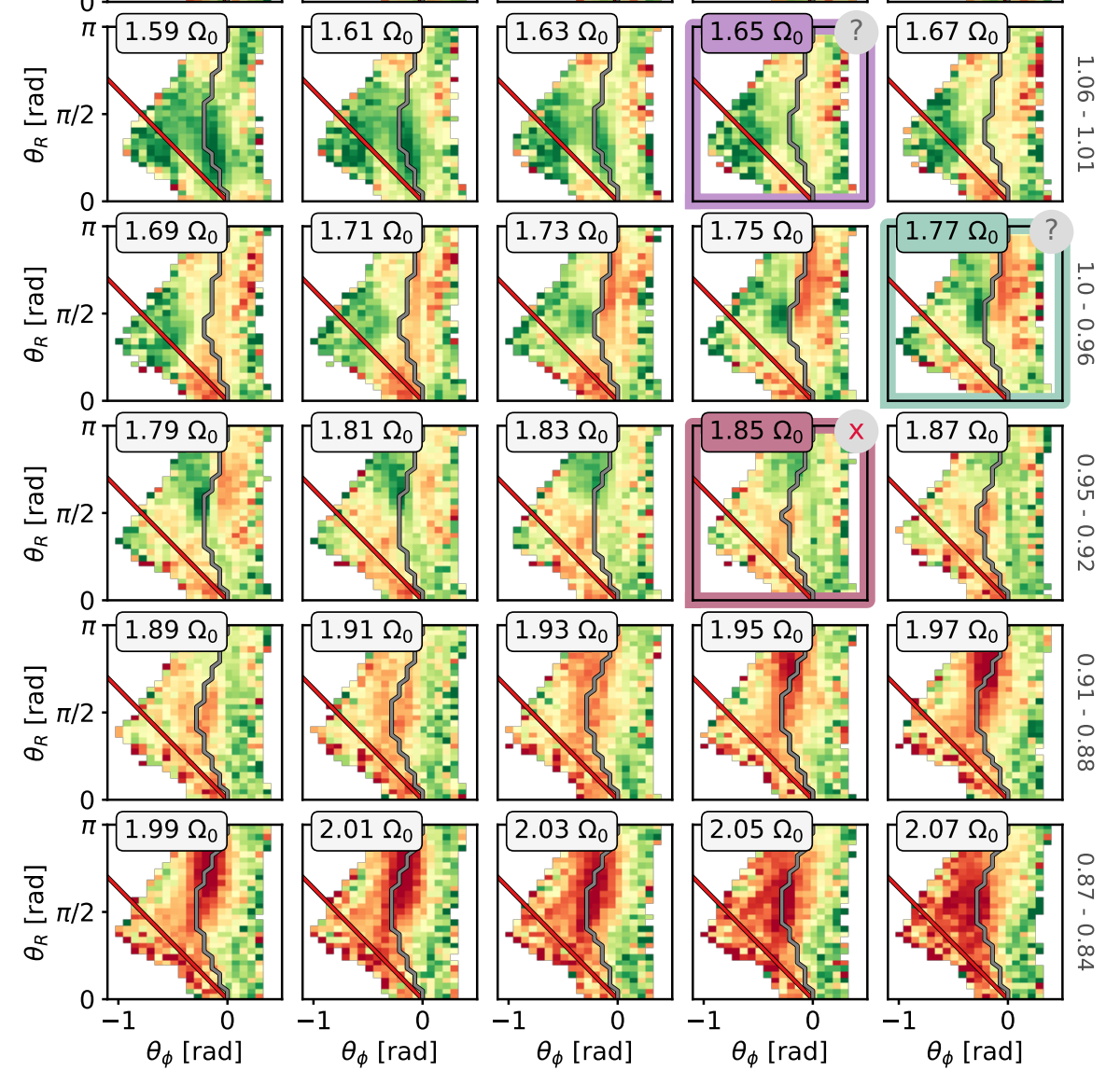

Figure D1. The Gaia action-angle data for the MW potential model by Eilers et al. (2019) (with $\Omega_{0}=28.3 \mathrm{~km} / \mathrm{s} / \mathrm{kpc}$ ), analogous to Figures B1 and 8. OLR candidates derived from the two methods are compared: (i) slope of -2 in angle asymmetry on the right vs. (ii) 'red/blue' feature at the OLR ALR on the left. The strongest candidate in angle space is also here close to the Sirius ridge, for $\Omega_{\mathrm{bar}}=1.45 \Omega_{0}$, similar to the slightly faster S19B19 pattern speed. 